\title{
Switch the Tumor Off: From Genes to Amanita Therapy
}

\author{
Isolde Riede ${ }^{*}$ \\ Independent Cancer Research, Im Amann, Uberlingen, Germany \\ *Corresponding author: riede@tumor-therapie.info
}

Received July 17, 2013; Revised October 25, 2013; Accepted October 29, 2013

\begin{abstract}
Tumor formation is due to somatic mutations. Mutant oncogenes and tumor suppressor genes lead to destabilization of the differentiation pattern and to defect cell adhesion. Proliferative genes are the cause of tumor formation, allow replication and thereby shortcut the cell cycle. At least one proliferative mutation is present in every tumor cell. Biochemical characteristics for tumor cells are onset of replication, constitutive onset of the repair system, and cell death defects. A successful tumor therapy inhibits specifically tumor cell activity and not the immune response. Thereby, the immune system is able to recognize and to digest tumor cells. In search of the center of the tumor pathways, switch genes are identified, that influence in trans tumor cell activity. Switch genes are not mutant, but overexpressed in human tumor cells. All switch proteins are RNApolymeraseII transcription factors. Therefore in tumor cells RNApolymeraseII should be used to full extent. Amanita phalloides contains amanitin, inhibiting RNApolymeraseII. Applying Amanita phalloides dilutions lead to reduction of the tumor cell activity, and in cancer patients to stabilization of the disease state, or eventually to remission.
\end{abstract}

Keywords: tumor formation, oncogenes, tumor suppression, proliferative genes, switch genes, Amanita phalloides, tumor therapy

Cite This Article: Isolde Riede, "Switch the Tumor Off: From Genes to Amanita Therapy." American Journal of Biomedical Research 1, no. 4 (2013): 93-107. doi: 10.12691/ajbr-1-4-5.

\section{Introduction}

It is generally accepted, that a tumor originates from cells with a somatic mutation. Proto-oncogenes are instrumental in developmental processes such as cell communication, signal transduction and regulation of gene expression [1,2,3]. A number of human cancers are associated with mutation or loss of a tumor suppressor gene. So far it was suggested that their protein products keep in check an otherwise uncontrollable ability of cells to proliferate $[4,5,6]$. Tumors in humans are rarely associated with single gene defects [7,8]. Two major features are altered in tumor cells. Firstly they lose control over the cell cycle and proliferate in an unregulated fashion. Secondly they lose or alter differentiation. Although general tumor suppressor genes are known $[9,10,11]$, their specific role in tumor formation had not been illuminated. Instead, it had been generally accepted, that the mutational alterations of oncogenes and tumor suppressor genes somehow activate cellular growth.

In general, every tumor carries its own set of mutations. Tumor cells show a defect in the program for cell death and resistance to chemotherapy. They show genome lability and infinite growth capability, loss of telomers. To clear the biochemical role of related genes, in a novel approach, a Drosophila genetic model had been developed to analyze general features of the genes. Experiments are summarized here, to give rise to a completely different view on cancer genetics: The proliferative event is the cause of tumor formation. It causes most of the defects known from cancer cells. Oncogenes and tumor suppressors only add to the differentiation patterns.

Tumor induction is due to a mutation in a proliferative gene that allows replication in Drosophila [12]. One single proliferative mutation is sufficient for tumor formation. But are the genetic and biochemical events in Drosophila comparable to tumor formation in humans? In a novel approach, the correlation of the initial biochemical events had been illuminated in human All-1 mutant leukemic cell lines [13]. In those cell lines, replication starts immediately after mitosis. All-1 mutations thus allow replication without control - defining All-1 as proliferative gene. As well as in Drosophila, one proliferative mutation allows replication, shortcuts to the S-phase to initiate tumor growth of cells. Therefore, tumor induction in humans and tumor induction in Drosophila is due to the same biochemical event. Therefore results from both systems are presented and discussed here consistently.

Drosophila genetics offers a system to identify genetic interaction and biochemical and cytological events within a reasonable time. The identification of the central molecular switches in tumor cells, are found by interactive genetics. Here, the identification of switch genes is summarized. The way to use the knowledge for the Amanita therapy is illuminated, and some therapy examples are outlined.

\section{Involved Genes and Functions}

\subsection{Oncogenes}


The early events during Drosophila embryogenesis, from the view of a single cell, involve a modulation of two features. First, the expression pattern of the genes active in the G1 or G0 phase, as well as the functions of the cell within the organism has to be defined. This type of modulation determines the cell fate and differentiates the tissue. Second, a programmed number of cell cycles proliferation - has to be driven. Differentiation and proliferation of cells require equilibrium. Excessive proliferation will lead to more cells than programmed, leading to hyperplasic growth. Premature differentiation of several cells, before the programmed number of cell cycles is driven, will lead to missing cells: organs or structures are incomplete. A lack of differentiation in addition with proliferation will lead to an overgrowth of undifferentiated tissue and, accordingly, to a tumor.

In Drosophila, the establishment of the basic features of the dorsal-ventral embryonic pattern requires specifically the action of maternal effect genes [14]. Signal transduction is triggered on the ventral side of the Drosophila embryo through the binding of an extracellular ligand to the Toll (Tl) receptor [15]. $\mathrm{Tl}$ encodes a transmembrane protein, homologous to the human interleukin-1 receptor [16]. spätzle (spz) acts immediately upstream of $\mathrm{Tl}$ in the pathway and represents a component of the extracellular signaling pathway $[17,18]$. Downstream of $\mathrm{Tl}$, the action of pelle (pll), a protein kinase, is required [19]. The activities of spz, Tl and pll result in a ventral to dorsal gradient of dorsal (dl) protein in the nuclei of the syncytial blastoderm of the embryo.
This gradient gives rise to region specific expression of zygotic genes. One of them, tolloid (tld), is transcribed by nuclei in the dorsal-most $40 \%$ of the blastoderm.

\section{Larval Brains: Tumors}

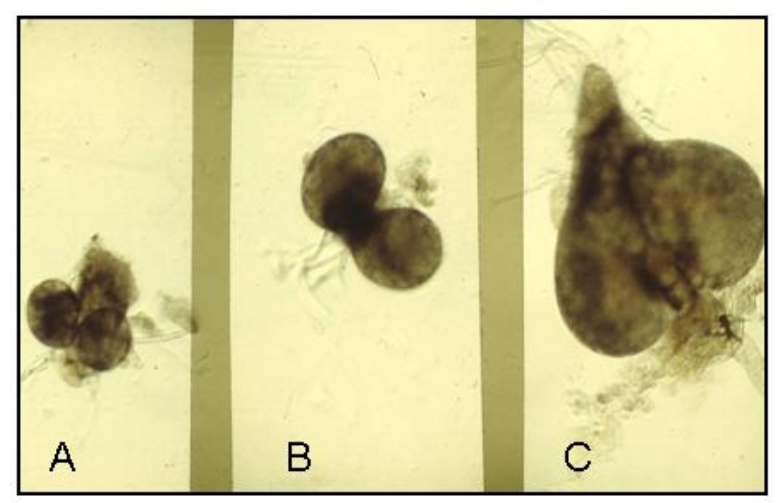

Figure 1. Malignant Brain Tumor is induced by several genes

The Drosophila melanogaster strain Malignant Brain Tumor (MBT) reveals temperature sensitive transformation of the larval brain tissue. Genetic analysis had shown that three gene defects, $s p z^{M B T}$ yeti ${ }^{M B T}{ } d^{M B T}$ cooperatively induce brain tumor formation. $s p z^{M B T}$ appears as an oncogene, $t d^{M B T}$ was identified as the tumor suppressor gene and yeti ${ }^{M B T}$ was found as the proliferative gene. A: Larval brain of wild type. B: Larval brain of homozygous Drosophila with yeti ${ }^{M B T} s p z^{M B T}$. C: larval brain with mutant genes höcker ${ }^{M B T}$ hedera $^{M B T}$ mali $^{M B T}$ tld $^{M B T}$ yeti ${ }^{M B T}$ $s p z^{M B T}$. All brains were obtained from stage three larvae, animals were kept at $29^{\circ} \mathrm{C}$. Light microscopy.

\section{Oncogenes}

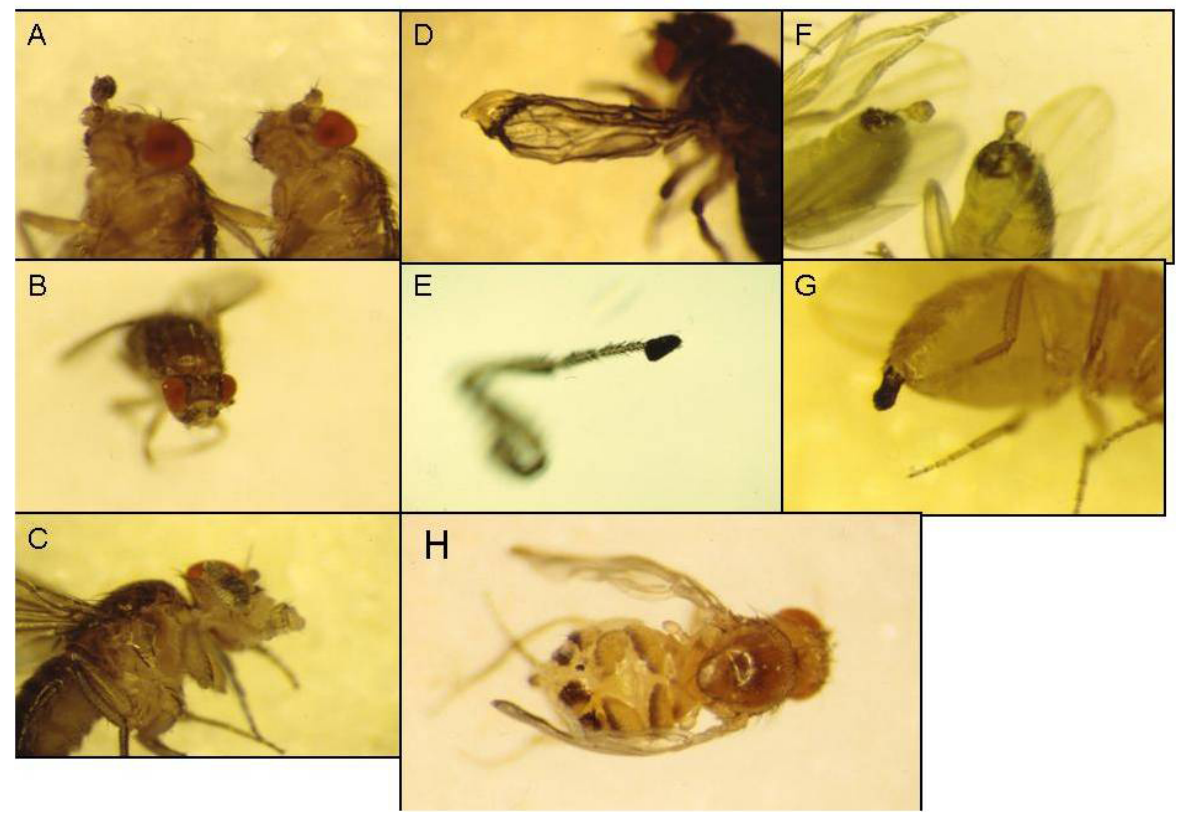

Figure 2. Oncogene Actions

Depending on the genetic combination, different tissues destabilize and eventually grow as a tumor. Tumor formation in these cases is individual, not all animals induce tumor formation. A. $\mathrm{pll}^{628} /$ yeti $^{M B T} s p z^{M B T}$ : antennae grow in asymmetry, and tumor tissue can grow due to the proliferative gene yeti ${ }^{M B T}$. B. $\mathrm{pll}^{628} / \mathrm{spz}^{\mathrm{MBT}}$ : due to asymmetric cell divisions, the right eye of this animal is larger than the left one. Thus, at a distinct time during development, more cell cycles run in one eye than in the other. No tumor is visible. C. Df(3R) $)^{\text {roest1] }} / s p z^{M B T} y e t i^{M B T} t l d^{M B T}$ : whereas homozygous $s p z^{M B T} y e t i^{M B T} t l d^{M B T}$ is larval lethal and develops a malignant brain tumor, animals with this genetic constellation develop to adults. The deficiency comprises the gene yeti ${ }^{\text {MBT }}$ in 96F. Tumor formation is active, neural tissue grows out of the eyes. D. pll ${ }^{628} / \mathrm{spz}^{M B T}$ yeti ${ }^{M B T}$ some of the flies fail to develop an extended wing. Cells are allowed to grow at this destabilized site. E. $\mathrm{Df}(3 \mathrm{R})^{8 \mathrm{D} 06} / \mathrm{spz}^{\mathrm{MBT}}$ yeti ${ }^{\mathrm{MBT}}$ : This genetic constellation allows development of adults. The deficiency comprises the gene yeti ${ }^{M B T}$ and Enhancer of split $(E(s p l))$. The tip of the leg is destabilized and grows as a tumor. F. bellatrix $/$ Df(3R) ${ }^{\mathrm{R} 1}$ : The deficiency comprises the gene yeti ${ }^{M B T}$ and $E(s p l)$, this constellation leads to overgrowth of the last segment. G. $N^{55 e 11} / \mathrm{mali}^{M B T}$ yeti $^{M B T} s p z^{M B T}$ leads to a necrotic overgrowth of the last segment. H. $s p z^{M B T}$ yeti $i^{M B T} / s p z^{67}$ shows the differentiation defect due to the oncogenic spz mutation. All animals were grown at $29^{\circ} \mathrm{C}$. Light microscopy. 
Proto-oncogenes have been identified by dominant (gain of function) mutations, and are involved in many cellular pathways. Drosophila mutant Malignant Brain Tumor (MBT) carries six mutations cooperatively inducing brain tumor formation (Figure 1, [20]). Both in humans and in Drosophila, malignant tumor formation can be due to a multigenic process. $s p z$ was identified being mutant in MBT, as oncogene. $\mathrm{Tl}$ and pll alleles induce tumor formation in trans over MBT (Examples in Figure 2, [21]). This identifies $T l$ and pll as protooncogenes. Human homologues of $\mathrm{Tl}$ are involved in many respects in human cancers [22,23]. In colon carcinoma the $T l$-like receptor pathway is deregulated in peripheral blood cells [24]. Therefore developmental genes of the dorsal-ventral patterning pathway are protooncogenes in humans and in Drosophila. spz, pll or $\mathrm{Tl}$ mutations alone or in combinations do not lead to malignant cell proliferation. Thus, oncogenes are not the cause of cell proliferation.

\subsection{Tumor Suppression}

Tumor suppressor genes add to tumor formation when their function is absent. Both copies of the gene on the homologous chromosomes have to be defect. Thus a recessive or loss of function mutation is involved. Tumor suppressor activity in MBT maps to $85 \mathrm{cM}$ on the third chromosome and was identified as $t d^{M B T}$ allele [20]. Rescue of animals, carrying homozygous 3rd chromosome, is possible with a wild type tld gene on a P-element on chromosome one or two (Figure 3). Transgenic MBT with addition of wild type tld partially rescue tumor formation: neuroblasts regain their ability to differentiate and lose malignancy. Still hyperplasia occurs, indicating that the proliferative event still is active, the tumor suppressor mutation only adds to tumor formation. $\operatorname{tld}^{M B T}$ alone, or in combinations with oncogene mutations does not lead to malignant cell proliferation. Therefore a tumor suppressor gene is not the cause of cell proliferation [21].

\section{Larval Brains: Tumor Suppression}
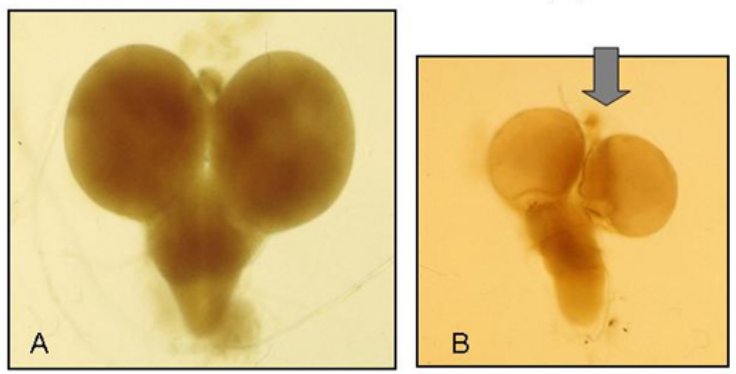

Figure 3. Tumor Suppression

At the restrictive temperature, MBT exhibits unsegmented optic lobes with undifferentiated malignant neuroblasts (A). $t d^{M B T}$ was identified as the tumor suppressor gene in MBT [20]. A plasmid that contained DNA encoding for the $3.5 \mathrm{~kb}$ transcript of tld+ on chromosome 1 or 2 were combined accordingly with the third chromosome of MBT (B). The enlargement of the brain is reduced by $t d^{+}$, the ventral ganglion is elongated. Ingrowth of tumor tissue into the ventral ganglion was not observed. Thus, cells have lost their ability to invade. The optic lobes still show hyperplasic growth. $t d^{+}$ transgenic MBT regain wild type pattern of ARD expression (arrow) in the brain, indicating that $t d^{+}$drives differentiation of neuroblasts. Animals were kept at $29^{\circ} \mathrm{C}$. Light microscopy.

Tld is homologous to Human Bone Morphogenetic Protein 1 [25]. The protein consists of an amino terminal metalloprotease domain followed by an interaction domain including complement protein repeat motifs and epidermal growth factor type three domains. Two domains of the protein contribute to its interaction with decapentaplegic (dpp), a member of the TGFß family [26]. This family of extracellular factors can stimulate or inhibit cell growth or differentiation, depending on the cell type involved [27]. Alleles of tld with known mutations show, that any allele being mutant in region around amino acid 250 shows strong interrelation in MBT. This region confers one of the tld-dpp interaction domains, within the metalloprotease region of tld. Thus it is anticipated, that the interaction of tld with dpp is important for the tumor suppressor function [21]. Human morphogenetic proteins, the human homologues of tld, are involved in human tumor aggressiveness [28,29,30]. Thus, tld is involved in tumor formation in humans and in Drosophila.

Mutations in developmental genes alone do not break the restriction of the cell cycle. Another class of genes is responsible for tumor growth of cells:

\section{Larvae, Proliferative Gene Defect}

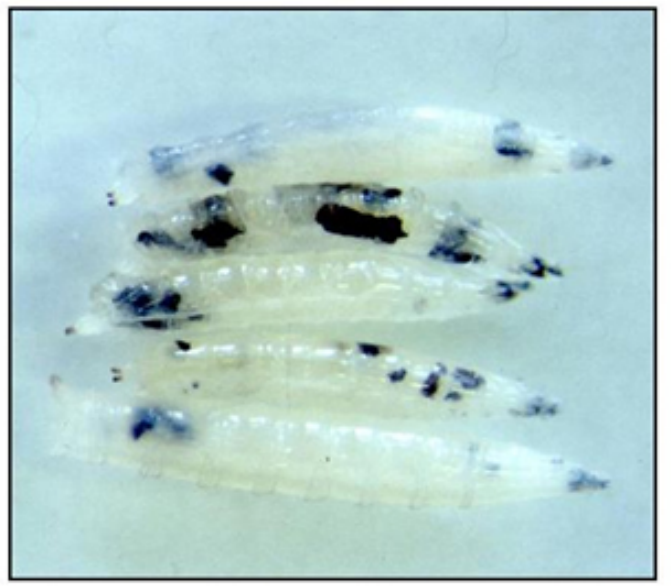

Figure 4. Proliferative mutation: melanotic lymphoma

All proliferative gene mutations in Drosophila show the same phenotype. They form a variety of melanotic lymphomas in third instar larvae [20]. Here homozygous mali $^{27}$, with only one proliferative gene mutation, are shown. Animals were grown at $21^{\circ} \mathrm{C}$. Light microscopy

\section{Proliferative Gene Defect: Lymph System}

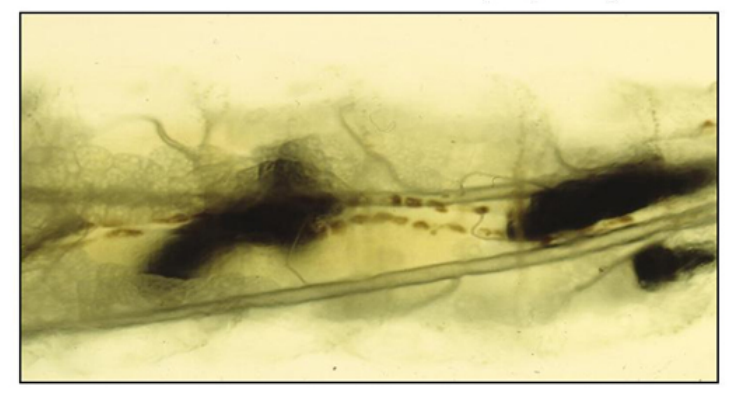

Figure 5. Proliferative mutation: malignant melanotic lymphoma

Homozygous mali $^{27}$ third instar larvae, with only one proliferative gene mutation, show melanotic tumor masses. The two lymphatics show melanotic cells as well in nodes. Thus, as in humans, the lymphatic system is affected from growing tumor cells. Thus, metastases occur with only one gene mutation. Animals were grown at $21^{\circ} \mathrm{C}$. Light microscopy.

\subsection{Proliferative Genes}

A proliferative gene mutation allows aberrant cell divisions. In the Drosophila multigenetic defect line MBT, the existence of a proliferative event was found in 96F1 
with yeti $^{M B T}$ [20]. Further analyses revealed that one single proliferative mutation leads to melanotic lymphomas (Figure 4). These melanotic lymphoma metastasize into the lymph system, like human lymphomas (Figure 5). Tumor formation in other tissue requires the addition of oncogenes and/or tumor suppressor genes mutations (Figure 2).

\section{Proliferative Gene Defect: Somatic Pairing}

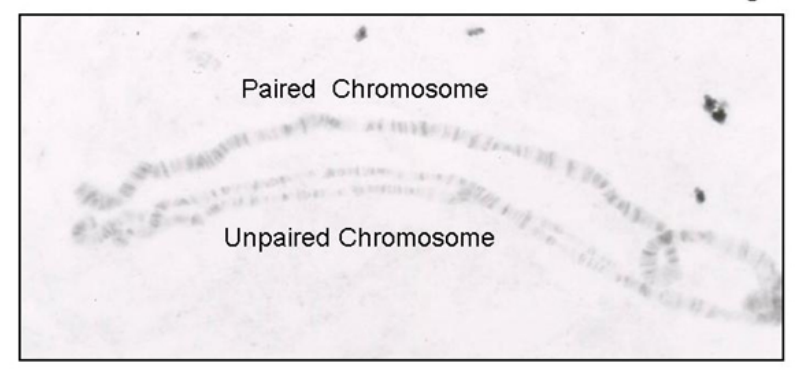

Figure 6. Proliferative mutation defect: somatic pairing

Proliferative Mutations induce somatic pairing gaps in salivary gland chromosomes of third instar larvae. This is a dominant phenotype. Here chromosomes of wild type/ merlin ${ }^{14}$ are shown. Animals were grown at $18^{\circ} \mathrm{C}$. Light microscopy of chromosome spread.

All proliferative mutations lead to pairing gaps of homologous chromosomes (Figure 6, [12]). In Drosophila, and presumably in all eucaryotic cells, homologous chromosomes are somatically paired. This pairing allows repair of the genome in case of double strand breaks with DNA recombination repair system. Vicinity of the homologous partner is a prerequisite for this. Chromosomes are paired during meiosis, to allow recombination. Proliferative mutations alter the frequency of meiotic recombination: in distinct regions, no recombination occurs, whereas in other regions increased recombination frequency is detectable. A structural component of the chromatin is involved.

\section{Proliferative Gene Defect: Genome Lability}

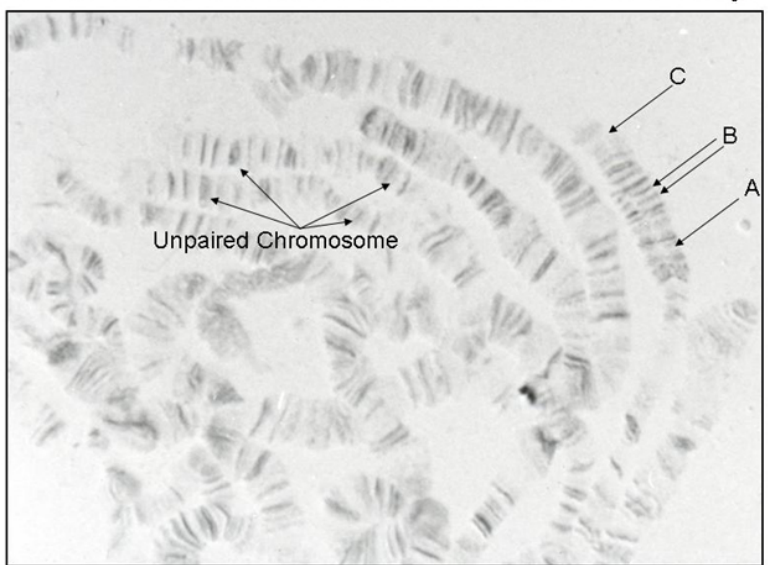

Figure 7. Proliferative mutation, unpaired chromosomes and genome lability

Nuclei in salivary glands of wild type/ bellatrix ${ }^{P}$ third instar larvae show fluffy chromosomes, not accordingly packed. Therein some chromosome regions are unpaired. In this case, pairing type 2 (A) occurs, incompletely paired chromosomes. Adjacent to this phenotype, asymmetries in replication occur (B), followed by a missing telomere (C). In case this phenotype occurs in dividing cells, genome instability is the result. bellatrix is a proliferative mutation due to a P-element insertion to vicinity of $T l$. Effects on chromosomes are dominant. Animals were grown at $18^{\circ} \mathrm{C}$. Light microscopy of chromosome spread.

Genome lability can be observed, leading to asymmetric replication of chromosomes (Figure 7). The involved proliferative mutation bellatrix ${ }^{P}$ is a P-element insertion to vicinity of proto-oncogene $T l$.

\section{Proliferative Gene Defect: Giant Chromosomes}

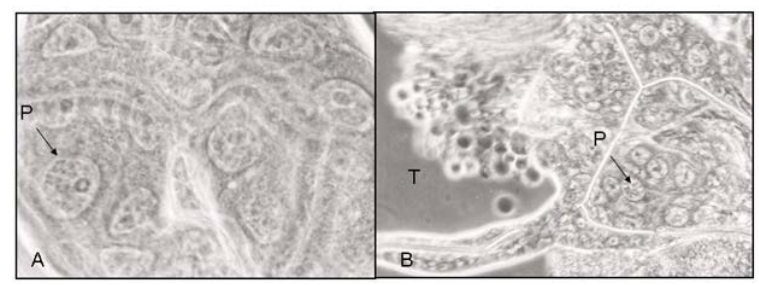

Figure 8. Proliferative mutation allows replication: giant chromosomes in tumor tissue

A. Brain tissue of homozygous $\operatorname{tld}^{M B T}$ yeti ${ }^{M B T} s p z^{M B T}$ shows areas with giant cells, obviously with polytene chromosomes (P). Wild type brain shows no polytene cells in the brain. Thus the biochemic origin of tumor formation, is correlated with allowance of replication. B. Within the tumor tissue (T) of homozygous yeti ${ }^{M B T} s p z^{M B T}$ third instar larval brain, polytene cells (P) occur. Animals were grown at $29^{\circ} \mathrm{C}$. Light microscopy.

\section{Proliferative Gene Defect: Giant Chromosomes}

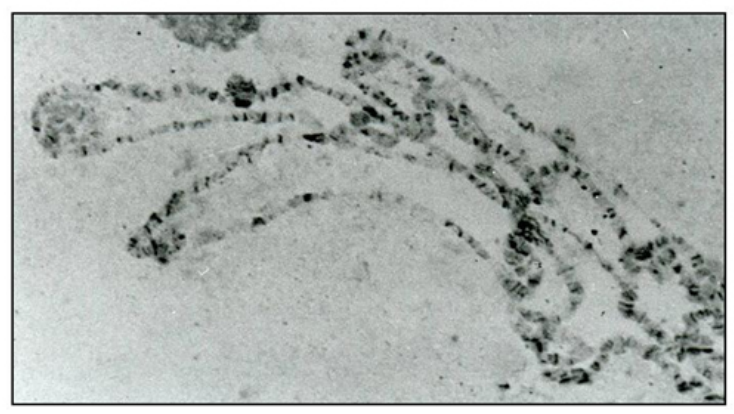

Figure 9. Proliferative mutation allows replication: giant chromosomes in brain tissue

Brain tumor formation is a polygenic effect, not due to a single mutation. The brain of all proliferative gene mutants is not overgrowing, but smaller than wild type brain. The third larval instar brain of homozygous hexe $^{77}$ was spread, many polytene chromosomes were identified. Thus, the allowance of replication is due to the proliferative gene defect. In wild type, no polyteny had been observed in brain cells. Animals were grown at $18^{\circ} \mathrm{C}$. Light microscopy of chromosome spread.

All proliferative mutations allow aberrant polytenization of cells (Figure 8, Figure 9). In polytene cells several rounds of replication occur. A proliferative mutation allows replication and in that shortcuts the cell cycle (Figure 10).

Proliferative alleles on chromosome 3 could be found and located to 24cM (teufel), 56cM (stern (srn), merlin), 84B (Antp), 87B (mali), 88cM (amanda), 96F (yeti) and 97F (efendi (efe)) [12]. Around ten loci per chromosome could represent proliferative loci. This is indicated in Figure 11 as red stars in the upper line. They do not carry repetitive DNA, but interfere genetically. Proliferative alleles at 56cM are strong: $A n t p^{A U S}, s r n^{88}$ and $m e r^{14}$ induce lethality and melanotic lymphomas in homozygous and over wild type heterozygous animals. In addition, srn induces lethality in trans over a majority of other proliferative alleles, defining srn as the central proliferative gene.

The genes interfere with each other in a quantitative matter. For example $e f e^{89}$ is a recessive proliferative allele, mutant in a gene at $92 \mathrm{cM}$ on the third chromosome far from $s r n^{88}(56 \mathrm{cM})$. efe $e^{89}$ does not complement $s r n^{88}$, all hybrids die as third instar larvae with melanomes. In Figure 11 the identified proliferative loci of the 3rd chromosome, and their interactions is summarized. 


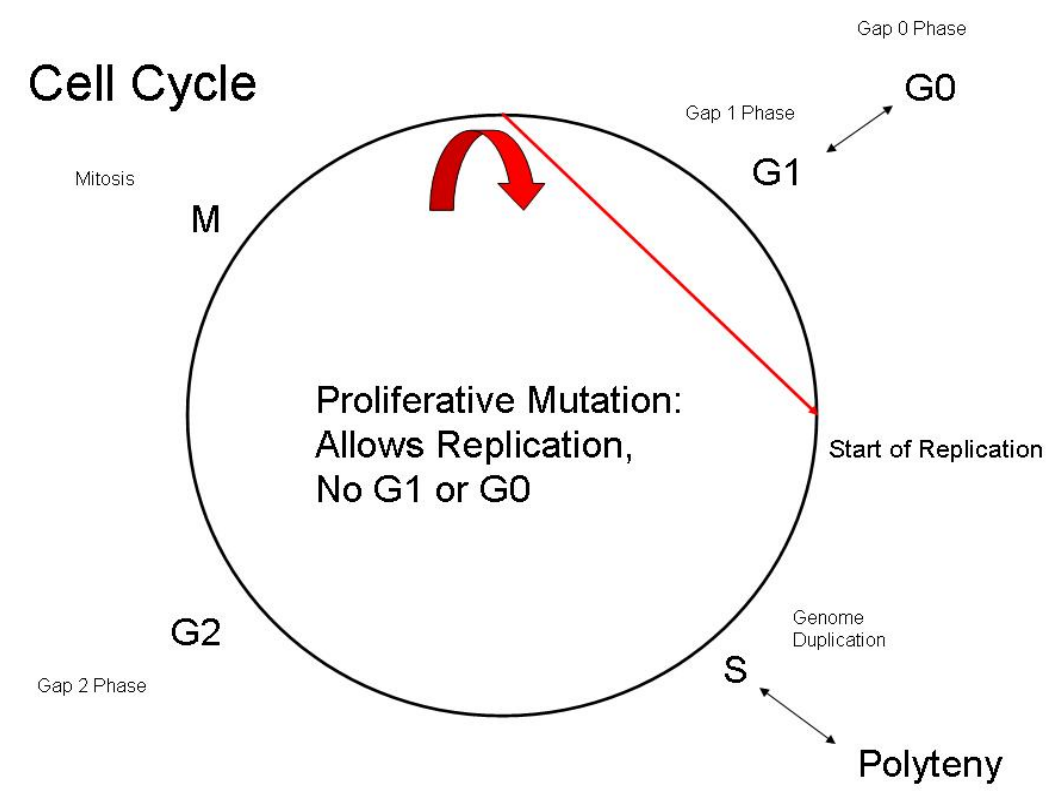

Figure 10. Cell Cycle with proliferative mutation

A proliferative mutation allows replication and thereby shortcuts to the S-phase, with no remaining possibility to restrict in the G1 or G0 phase.

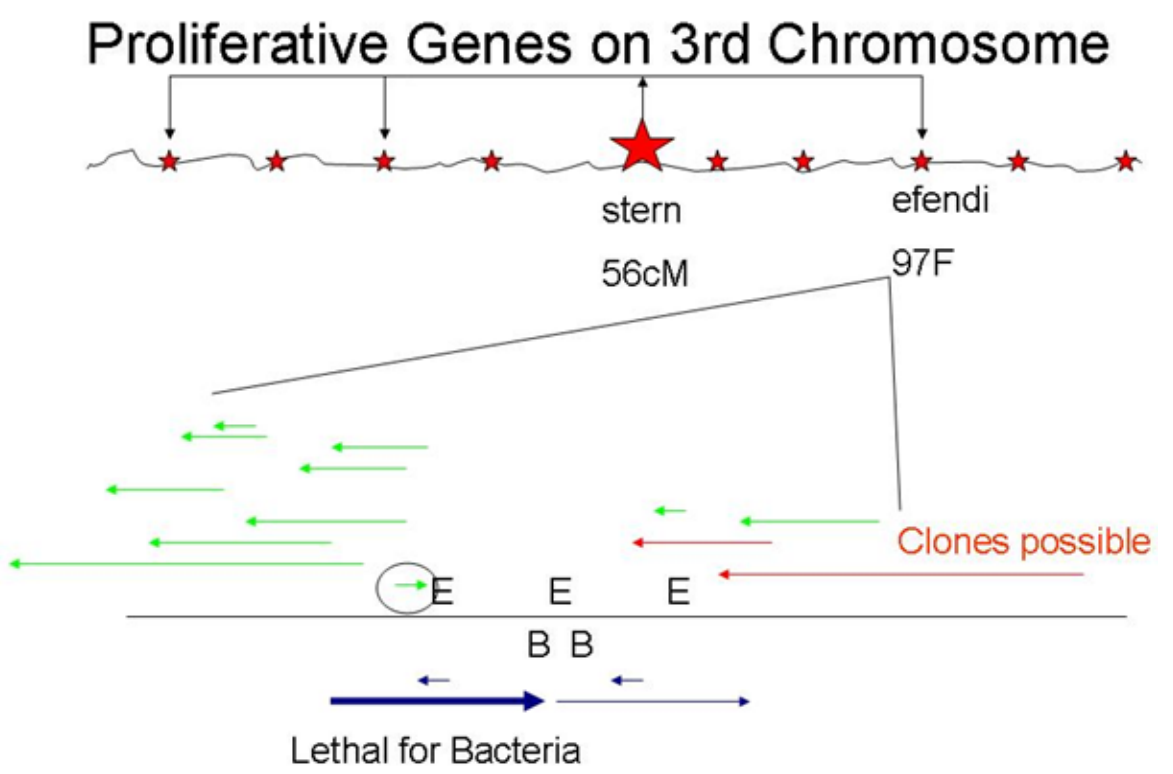

Figure 11. Proliferative Genes on third chromosome of Drosophila

The upper line represents the third chromosome, identified loci with proliferative genes are indicated as star. Gene srn is marked as a big star, this gene interferes in trans with most other genes, and is seen as central switch. Gene efe has been due to molecular analysis. The black line represents the genomic DNA, given are EcoRI (E) and BamHI (B) restriction sites for orientation, around 23.091.400 in the Drosophila genome in 97F1. Upper arrows represent the poly-A transcripts identified by northern blots. They are only marked schematically, not located exactly. The region is transcribed intensely during all stages of development, dominant direction of transcription is telomer to centromer. One $0,9 \mathrm{~kb}$ poly-A transcript is found centromeric to telomeric (circle). In c-DNA libraries, two of these transcripts can be found (red arrows, in the libraries called l(3)mbt). No other transcript appears in libraries. Thus, all other transcripts are suspicious to be lethal for bacteria. The blue arrows show the identified hot spots for lethality, with the most lethal direction of transcription. Lethality is highest, when DNA is transcribed in the direction of the small antisense transcript the circle.

Whereas EMS induced proliferative mutations (i.e. missense mutations, leading to aminoacid exchange in the proteinproduct) lead to melanotic tumor formation, none of the obtained P-element insertions into proliferative genes induces melanotic tumors. However, all P-element insertions destroy the somatic pairing in certain chromosome regions. Therefore, for tumor induction a misled gene product is required. Misled activity of the gene product leads to a tumor, destruction of the gene confers lethality, not tumor formation [31].
One proliferative locus, efe, was analyzed by molecular biology. The adjacent region is transcribed to about seven different poly-A transcripts. Out of them, only two can be cloned as c-DNA. All other transcripts fail to appear as clones, thus are suspicious to be lethal for bacteria (Figure 11). The related genomic DNA can be cloned, but Escherichia (E.) coli carrying this DNA show poor and abnormal growth. To illuminate the cellular function of the proliferative genes, the phenotypic manifestation in $E$. coli under sublethal condition was analyzed (Figure 12). 
Electron microscopic evaluation revealed, that the bacterial genome is not ordered as one or two light spots in one bacterium, but spread in several smaller spots throughout the bacterium. Bacteria are hyperlong, thus cell division is affected - a phenotype similar to recA mutants [32]. Bacteria with DNA from the wild type proliferative gene efe duplicate their genomic DNA without coupled cell division.

\section{Proliferative Transcripts: Lethal For Bacteria}
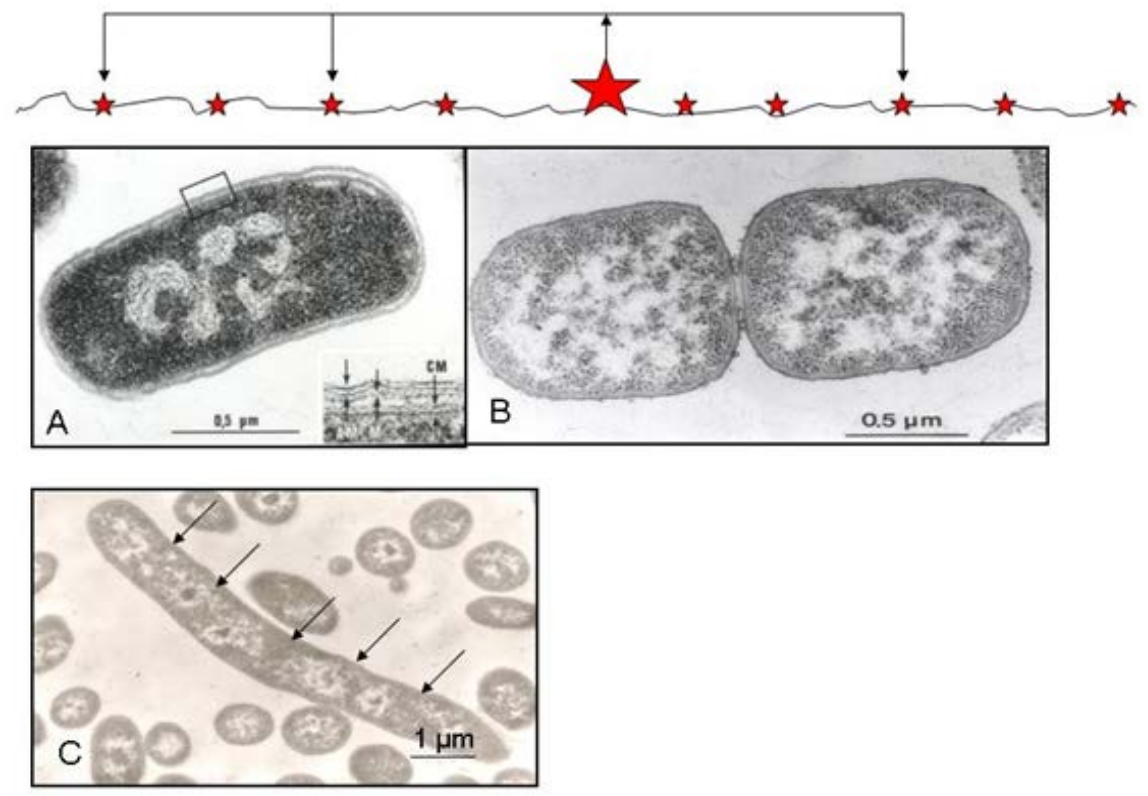

Figure 12. Proliferative Gene: ubiquitous action

A. Normal E. coli cell with one genome (light area) within ribosomes (dark spots). B. Normal dividing E. coli C. E. coli with recombinant DNA of efe, i.e. the 4,1 kb SalI-BamHI fragment in pKS (most lethal transcription as indicated by the blue arrow in Figure 11). The genome has duplicated several times without cellular division. At the sites of the arrows, cell divisions are expected. Bacteria are hyperlong, growth is sublethal. Bacteria were grown at $22^{\circ} \mathrm{C}$ over days on agar plates. Electron Micrograph.

The recA protein is involved in prokaryotic recombination and DNA repair mechanisms. It catalyzes pairing and strand exchange between homologous DNA molecules. In mammals, seven homologues, known as the Rad family were identified [33]. In Drosophila one stringent homologous gene is known [34]. Both in situ hybridization and Southern analysis indicate that the recA/Rad51 homologous gene is present only at 99D on chromosome arm 3R. Phenotypic manifestations of proliferative genes and recA are related: They are involved in chromosome pairing and recombination, in bacteria similar phenotypes are induced by both. However, no proliferative gene could be identified so far to vicinity of 99D. Therefore $\operatorname{rec} A$ is not identified as a proliferative gene.

\subsubsection{All-1 is a Human Proliferative Gene}

The Drosophila gene trithorax (trx) is related to the human All-1 [35]. All-1 mutations participate in acute leukemia in $5 \%$ to $10 \%$ of children and adults. Like other genes, All-1 was discovered by the association with chromosome rearrangements, involving its location in chromosome band 11q23 [36]. Molecular analysis has revealed a mutation called "self fusion". Those mutations that lack duplicated zinc finger motifs are associated with trisomy 11 [37]. Thus, mutation of the gene might be involved in allowing replication of chromosome 11. Like proliferative genes, tumor formation of All-1 is due to a gain of function of a new gene product. Only fused functional genes are involved in leukemia formation, destruction of the gene are not identified in tumor cells.
Is All-1 a proliferative gene? Cells usually enter a G1 (gap 1) phase after mitosis (Figure 10). This is the regulation phase for each cell type, and is the time at which most biochemical functions are executed. Subsequently, the cells may enter a G0 phase, meaning that the cell reaches its final differentiation, with no remaining ability to divide. This modulating phase is shortcut by a proliferative mutation, which allows replication without restriction. After the $S$ (synthesis of DNA) phase, cells go through a short G2 phase, which cannot be shaped, but is fixed in time and biochemical action to prepare the cell division ( $\mathrm{M}$, mitosis). Proliferative genes allow replication, this leads to the Sphase directly, to polyteny or giant chromosomes, and dedifferentiates the cell, with no restriction point to arrest the tumor cell. If All-1 mutation is a proliferative gene defect, cells should allow replication without regulation. Aberrant onset of replication would be expected directly after mitosis, when G1 phase should start.

In Figure 13 several replication profiles of synchronized leukemic cell lines are shown. In K562, with wild type All-1, some DNA synthesis starts after 9-11 hours, S-phase occurs 15-19 hours after release of the cells. This is followed by G2 phase, with no further replication. Cells with All-1 translocations show a different profile: Immediately after mitosis replication starts, is allowed. A G1 phase never occurs, and no S-phase with following G2 phase is visible, not within one cell cycle, nor within a period of 40 hours. Thus, All-1 is a proliferative gene, allowing replication. 


\section{Human Proliferative Gene All-1}

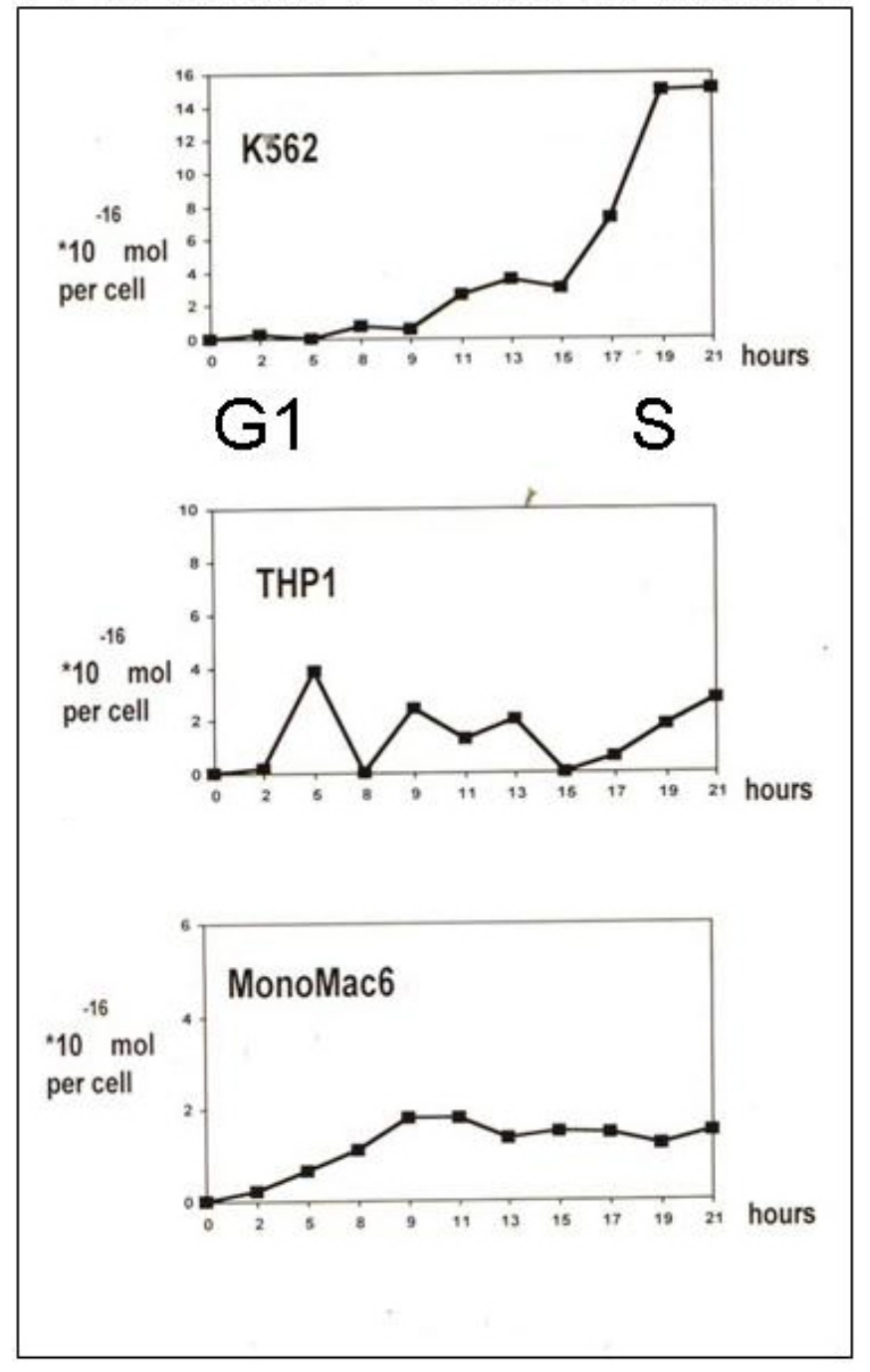

Figure 13. Human leukemic gene All-1 is a proliferative gene

Shown is the 3HT incorporation into DNA in synchronized cells [13]. Cells end mitosis at time 0, thus G1-phase starts (see Figure 10). K562 is a leukemic cell line with wild type All-1. After some time, incorporation of 3HT starts, and the S-phase can be identified at about 17 hours, followed by the G2 phase and mitosis. THP1 and Monomac carry All-1 transformation. DNA incorporation starts immediately after mitosis, therefore All-1 is a proliferative gene mutation, allowing replication.

\subsubsection{Apoptosis and Programmed Cell Death Defect}

In humans, tumor formation is frequently coupled with apoptosis defects. Apoptosis is a cellular program, which induces an active self destruction of the cell, by activating RNases, DNases and Proteinases [38].

\section{Embryos: Programmed Cell Death}

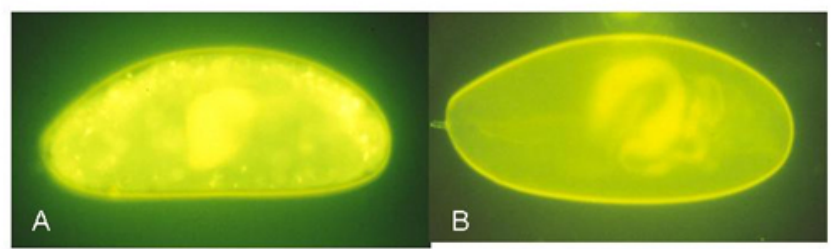

Figure 14. Programmed Cell Death Defect

A. During development, in wild type embryos more cells appear especially in the neuronal system than are needed. In late embryogenesis, these dispensable cells die, by programmed cell death. Here an embroy stained for 2 minutes with acridinorange is shown. Acridinorange is intercalating into the DNA, and marks dying cells with DNA fragments. Intact cellular DNA does not stain. B. MBT develops a brain tumor in third instar larvae. In MBT embryos, programmed cell death is absent. Shown is a 5 minutes stained embryo. Animals were kept at $29^{\circ} \mathrm{C}$.
In Drosophila, programmed death in the developing nervous system serves to remove obsolete neurons and to adjust neuronal number [39]. It was observed, that in tumor forming stock MBT the program for cell death induction during embryogenesis is defect (Figure 14). In addition, in neuronal tumor tissue of third instar larvae, apoptosis resistant cells occur. In any tumor tissue, cell structures are visible after oxygen deprivation, meaning that the program for self destruction is defective (Figure 15).

Apoptosis can be induced by chemicals, which destroy the structure of the DNA like methane sulfonic acid methyl ester (MMS). This drug is used in human tumor therapy. In case of high DNA damage, cells should undergo apoptosis, tumor cells should lyse. Flies should die from cell destruction. However, flies that carry proliferative mutations, frequently are resistant to the drug. This resistance is intrinsic, no prior treatment of the animals could have induced it. By mutation of a proliferative gene, resistance to chemotherapeutic drugs occurs [31]. 


\section{Brain Tissue: Apoptosis}

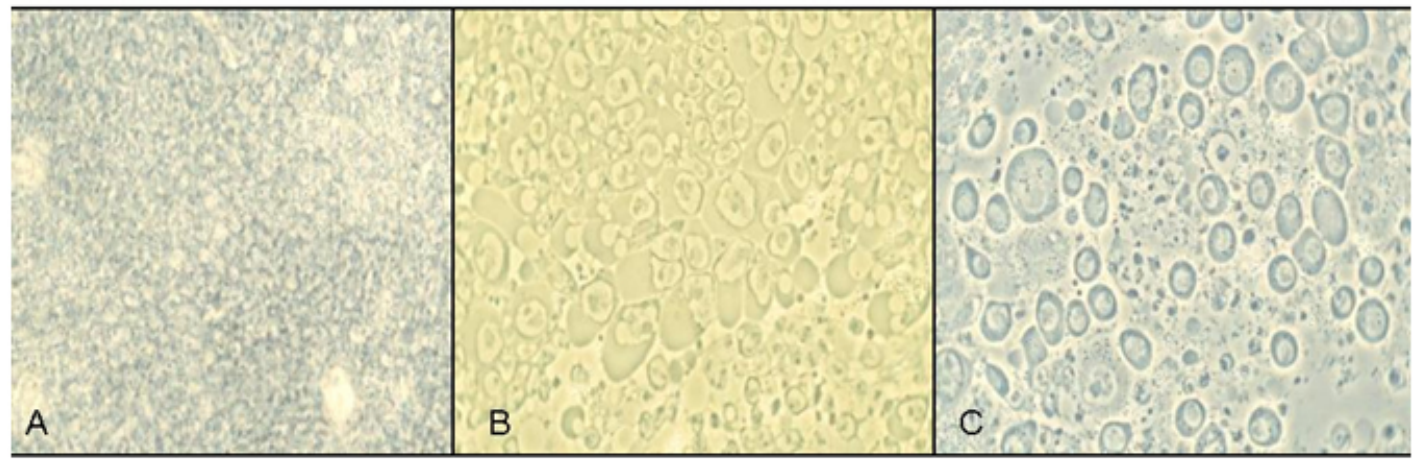

Figure 15. Brain tissue shows apoptosis defect cells

A Third instar larvae with homozygous yeti ${ }^{M B T} s p z^{M B T}$ mutation develop a hyperplasic non malignant brain tumor. Brain tissue taken from yeti ${ }^{M B T}{ }^{M p z^{M B T}}$ third instar larvae show after short time apoptosis of cells: Cellular structures lyse by active degradation with cellular digestion enzymes. Here tissue after 1,5 hours oxygen deprivation is shown. B Third instar homozygous MBT develop a malignant brain tumor. Tissue from this tumor contains cells, that undergo apoptosis, and cells, that do not activate this cellular program for cell destruction. Shown is tissue after 1 hour (B) and after 1,5 hours (C) of oxygen deprivation. Cellular structures still are visible.

\section{Central Switch to Apoptosis}

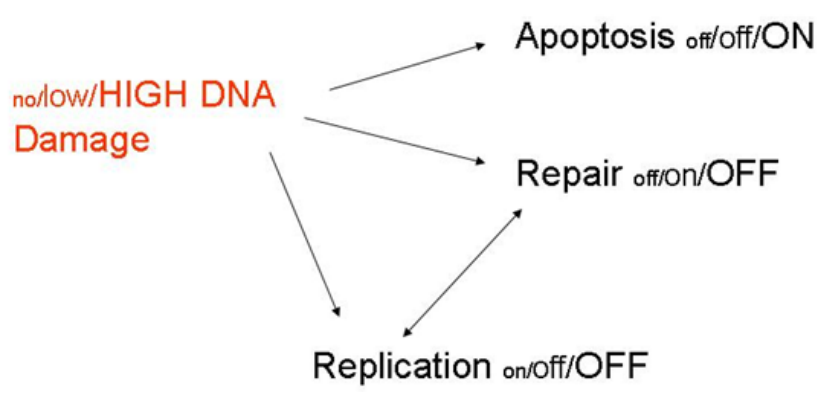

Figure 16. Cells decision way to Apoptosis (Explanation in main text)

In human cells carrying the All-1 mutation, repair replication is always active [13]. How do the cells react upon DNA damage to induce apoptosis? The central switch to apoptosis was analyzed by using the experimental model shown in Figure 16. This sheme predicts the regulation of the replication system, in case of DNA damage, to keep the genome intact. If there is no DNA damage, apoptosis should not be necessary; the repair system is off and replication is possible. If there is low, i.e., repairable DNA damage, apoptosis is off. One signal should stop replication to avoid establishment of mutations. One signal should induce, possibly via p53, the repair system [40]. If repair is successful, offset of repair and onset of replication follow. If severe DNA-damage is induced, like a number of double strand breaks of both homologous chromosomes that physically hinder recombination repair, replication and repair should stop. One signal to p53 should raise the apoptotic cascade. Herein, DNA damage leads the process, and a restriction point excludes both repair and replication at the same time.

DNA-damaging agents like MMS, Cisplatin or Bleomycin induce apoptosis in normal cells. However, all cells that carry the All-1 mutation are unable to induce apoptosis, instead they replicate and repair their genomic DNA (Figure 17 [13]). They cannot recognize DNA damage. In humans and in Drosophila, proliferative mutation is coupled to apotosis defect.

\section{Proliferative Mutation Excludes Apoptosis}

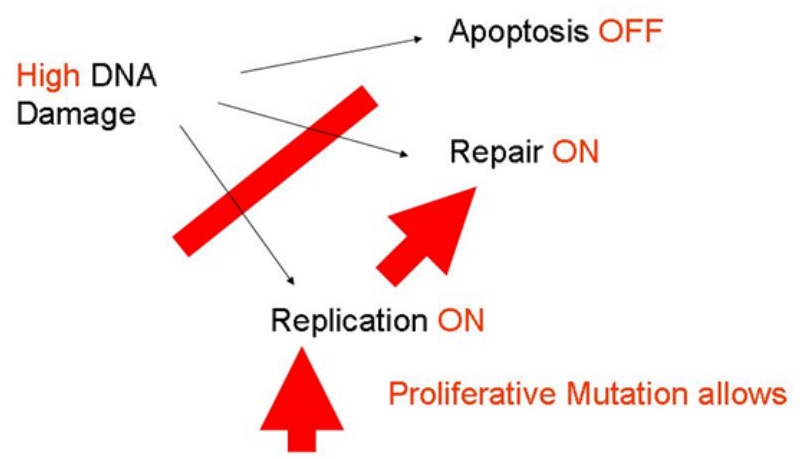

Figure 17. Proliferative Mutation Proliferative mutation excludes apoptosis

Whereas in a normal cell, DNA damage leads the repair system, All-1 mutant cells show onset of replication, and onset of repair. This constellation never occurs in normal cells, and could arrest the way to apoptosis. The repair system is now guided by the irregular replication and cells cannot recognize DNA damage. Onset of replication and onse of repair excludes onset of apoptosis.

A proliferative mutation allows replication, shortcuts to the S-phase. The repair system is involved in tumor formation, and many tumor cells cannot be brought to apoptosis induction. Mutational translocations within the gene All-1 that cause human acute leukemia act similar than the proliferative gene mutations in Drosophila: replication starts immediately after mitosis. Cells metabolize their DNA continuously. The repair system is constantly active. Induction of apoptosis fails. Thus, chemotherapy resistance is intrinsic, like in proliferative mutants of Drosophila.

One dominant proliferative mutation is responsible to

- allow replication

- shortcut the cell cycle

- allow tumor growth of cells

- allow metastatic behavior of cells

- show somatic pairing defects

- show recombination irregularities

- induce genome lability

- induce loss of telomers

- induce the repair system 
- induce apoptosis defect

- induce intrinsic resistance to chemotherapeutic drugs.

Tumor therapy with molecular tools requires central targets. Unfortunately on the level of proliferative genes molecular structures are complex, a central molecular event that could be inhibited by molecular interference is not visible. Therefore a molecular tumor therapy on this level is not visible. But the finding, that at 56cM strong interacting mutations could be found, that interfere with a number of other proliferative genes, oncogenes and tumorsuppressor genes, made the definition of another gene class possible:

\subsection{Switch Genes are Cental Interactors}

Switch genes had been identified by Drosophila genetics, that are able to influence in trans the tumor cell activity [41]. With a mutation in a switch gene, the characteristic trait of a tumor can be switched on or off. Usually, they are not mutant in tumors. Mutation of a switch gene is dominant lethal over a tumor mutation in trans, but permissive for wild type. Therefore, interaction on this level is lethal for tumor cells, and permissive for normal cells. Switch genes therefore serve as targets for novel drugs.

The genetic interlink between the proliferative genes and the signal transduction cascades can be identified, by crossing proliferative alleles over alleles in differentiation genes and screening the hybrids for lethality and growth aberrations. Alleles interfering were crossed over proliferative alleles. $s r n^{88}$ reveals as a major interactor.
Full lethality occurs over alleles of spz, Tl, cact, Serrate (Ser), and gro. Partial lethality was observed over pll, tub, and $d l$ alleles. $s r n^{88}$ does not complement efe alleles. As $s r n^{88}$ interferes with differentiation genes and with proliferative genes, it represents the genetic link between both. Interacting genes had been identified, and the genetic pathways are summarized in Figure 18.

Antennapedia (Antp) and Ultrabithorax (Ubx) interact with srn. srn and Antp thereby act as antagonists [41]. Switch genes show some phenotypic manifestations like proliferative genes: in few cases they form melanotic tumors, like srn. Their recombination frequencies vary. Switch gene alleles were analyzed for recombination frequency between $r i$ and $e$. $U b x^{79}$ hinders any recombination in this chromosome region. bicoid $(b c d)^{E 2}$, trx ${ }^{B 11}$, Polycomb ${ }^{9 M 2}$, Antp ${ }^{B 4}$, Antp $p^{73 b}$, Antp ${ }^{73 b+R 40}$, and $A n t p^{B}$, all increase the recombination frequency in this region by about $50 \%$. Thus, the switch genes act, on a similar biochemical level like proliferative genes, in the chromatin structure - changing recombination frequencies. Several switch genes show additional proliferative actions: Antp mutations form legs instead of antennae. Two features are involved in development of an animal: differentiation of cells and the defined number of cell cycles require equilibrium. To form a leg, more cell cycles have to occur, the organ is bigger. Thus, Antp is involved in supporting this equilibrium, a mutation can induce more cell cycles.

\section{Genetic Pathways in Drosophila}

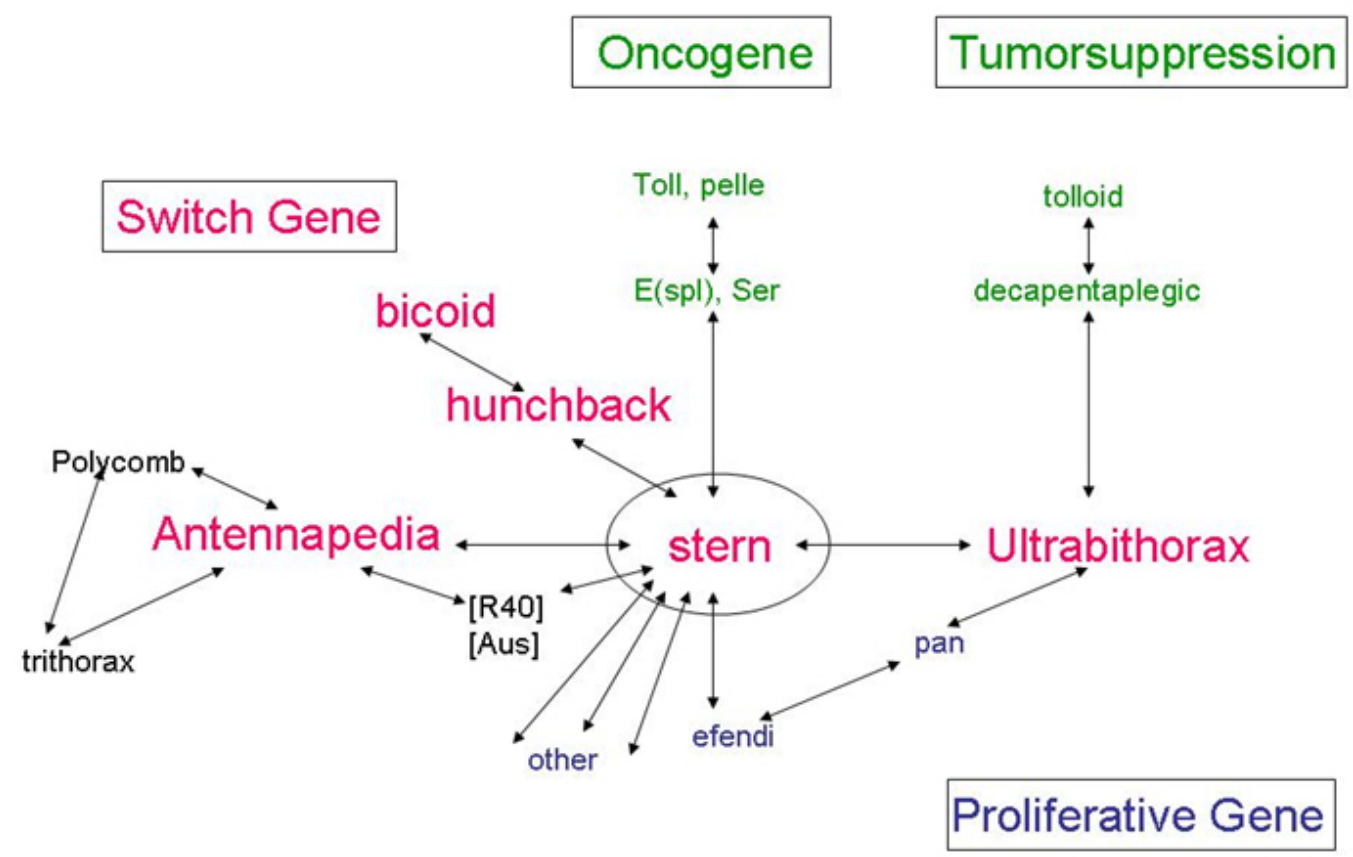

Figure 18. Gene interaction profile identified by Drosophila genetics

Some genes interact (arrows) with other genes in trans, meaning a hybrid fly with two recessive mutations in two different genes, which should appear as wild type, is not viable. Many mutations in proliferative genes interact with many other mutations in other proliferative genes [12]. Some - decentral mutations do not interact with many other mutations in other genes. Some - central - mutations interact with many other genes mutations. The central acting genes are Switch genes (red). The identified oncogenes (green) E(spl) and Ser produce incomplete wings when mutant, meaning that they promote cell cycles as wild type alleles.

A molecular tumor therapy, interacting on the level of RNA or DNA, does not open up. All switch genes show complex transcription/splicing patterns. All switch genes

carry the HOX motif. Antp, Ubx, bcd and hunchback (hb) belong to RNA-polymerase II (RNAPII) transcription factors and bind to DNA with specific motifs (Figure 19). 


\section{Biochemic Pathways in Drosophila}

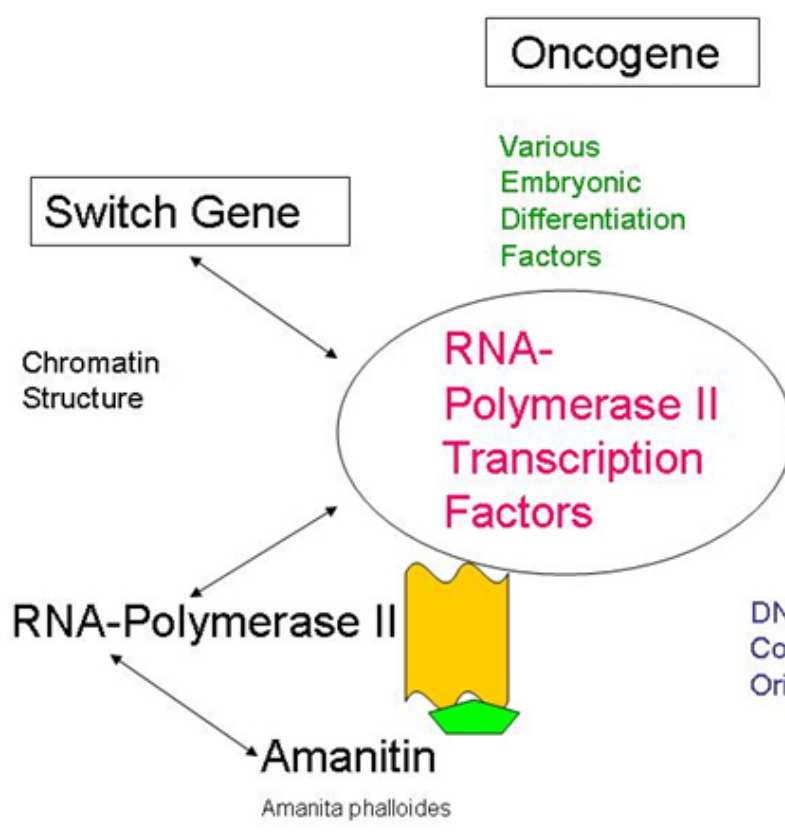

\section{Tumorsuppression}

DNA Binding Motifs,

Complex Gene Structures,

Origins of Replication?

Transforming

Growth Factor

Family TGFß

Figure 19. Biochemical pathways in tumor induction and tumor Acitvity

Biochemistry of the involved components of Figure 18: Oncogenes code for a large variety of factors, that are involved in differentiation patterns of cells during ontogenesis. Components vary from DNA-binding proteins, over membrane components, phosphatases or proteins of the lipid metabolism. All oncogenic components act together and are active in tumor cells as well as in normal cells. Tumor suppression is dominated by genes coding for proteins of the TGFß family. As well this family is involved in differentiation patterns of cells, and active in both tumor cells and normal cells. Proliferative genes show DNA binding motifs, and a complex molecular structure. Specific and successful molecular interference to inhibit tumor cell activity on these levels is not visible. Switch genes are complex and variable on the DNA- and transcriptional level. They modulate the chromatin structure and define the variety of genes that are imprinted to be transcribed. Downstream they interfere each with some 50 other genes. In human tumor cells, switch genes are overexpressed. All Switch genes are RNAPII transcription factors.

\section{The Amanita Therapy}

Switch genes are overexpressed in human tumor cells [42,43], (Figure 20). Therefore switch genes upregulate the tumor cell activity by overexpression. In that, many switch proteins act in the cell, that use the RNAPII to switch the tumor on. Therefore, the RNAPII is estimated to be used to full extent in tumor cells. This is one of the biochemical bottlenecks in tumor formation. Partial inhibition of this enzyme should break the activity of the tumor cell, will switch the tumor off (Figure 21). Amanitin, an inhibitor of RNAPII, is able to reduce tumor formation in Drosophila [44].

\section{Tumor Cells use RNA Polymerase to $100 \%$}

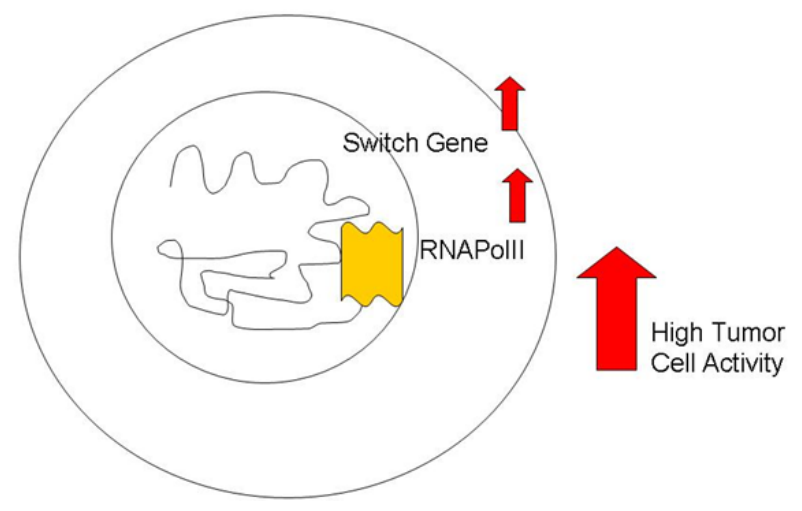

Figure 20. Switch Genes are overexpressed in Tumor cells and use RNAPII to full extent (Explanation in main text)

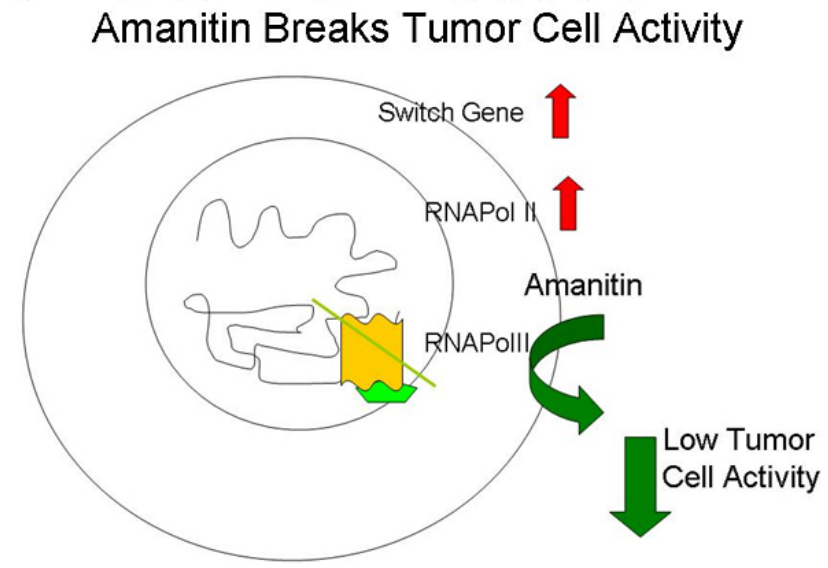

Figure 21. Amanitin binds to RNAPII and hinders switch gene action. (Thesis in main text)

Amanitin is not allowed today to be applied to humans, a new drug development is required. Amanita phalloides dilutions, that contain amanitin, are applied since 300 years, the classical homoeopathic indication is fear of death.

Partial inhibition of the RNAPII will not interfere with normal cells. The immune system is not inhibited by the therapy. With Amanita phalloides, the activity of tumor cells is reduced. They can be recognized by the immune system and are digested. In that, the Amanita therapy is not chemotherapy. The used concentration of the drug is low, the regimen of the therapy leads to about 7000 molecules applied per cell. The target RNAPII occurs in 15,000 molecules per cell. This is not lethal and will not 
induce apoptosis. It switches the tumor specific activity off.

\subsection{Therapy Example: B-Cll Stabilization}

Dilutions of Amanita phalloides, containing amanitin, are applied to a B-CLL leukemia patient (Figure 22, [44]). The patient was born in 1957 and works in the field service. B-CLL was first diagnosed in 2005, appearing slowly progredient. Amanita therapy started end of 2007 at a cell count of $1,26 \times 10^{5}$ leukocytes p.m. (per microliter blood). This leukocyte level is too low for conventional treatment. The patient decided himself to undertake a therapy attempt with Amanita, until a level of $2 \times 10^{5}$ leukocytes p.m. would be achieved or until side effects like liver cell damage would appear. In parallel all prearrangements for conventional therapy occurred. Anamnesis leads to no specific risk for tumor formation. Body weight is $77 \mathrm{~kg}$ and remains constant throughout the therapy. No additional disease was diagnosed. He subsists on vegetarian food and often suffers from low iron levels. He shows no symptoms of leukemia, no lymph node swelling, no frequent infections. The erythrocyte cell count is at $4 \times 10^{6} \mathrm{p} . \mathrm{m}$. at low level within the normal range.

No other tumor specific therapy is applied. Adjuvant uptake of additional essential fatty acids (to enhance fluidity of cell membranes and to provide raw material for prostaglandins, i.e. immune cell hormones), iron (orally) and zinc (cutanous) is applied. Noxes, i.e. long flights or radiation are avoided as far as possible.

\section{B-CLL Patient, First Three Years}

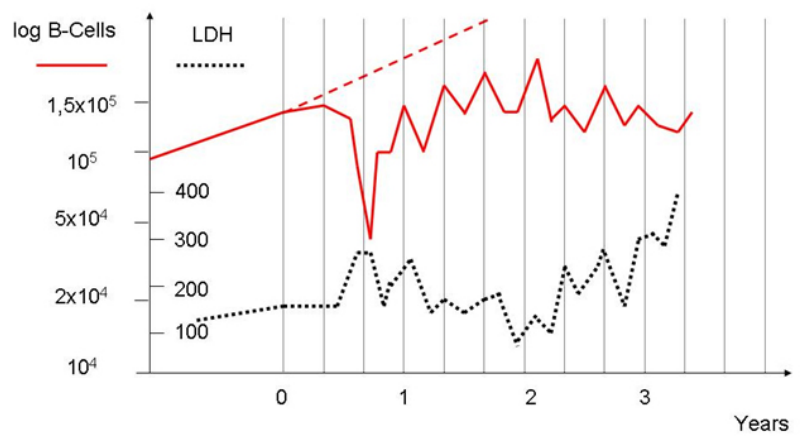

Figure 22. Stabilization of B-CLL leukemia

Outlined are the values of the leukocyte cell count in blood (log B-Cells p.m.) over time. Before Amanita therapy, the cells show exponential growth with a duplication time of 21 months (in graph broken red line). At time zero, Amanita therapy starts. LDH values (black dotted line) alternate with leukocyte count. Whenever a higher dose Amanita is applied, leukocyte values drop and $\mathrm{LDH}$ values rise revealing degradation of tumor cells.

Dosage:

0-3 months 3 x 15 drops D4 per day, equivalent to $7 \mathrm{ml}$ D2 incorporated. 4-7 months 4 x 10 drops D2 per day, in sum $82 \mathrm{ml} \mathrm{D2} \mathrm{incorporated.}$ 8-10 months break of 5 days, followed by 5 x 15 drops D4/d, in sum 95 $\mathrm{ml}$ D2 incorporated.

11-12 months 4 x 10 drops D2 per day, in sum $130 \mathrm{ml}$ D2 incorporated. 13-15 months break.

16-17 months 4 x 10 drops D2 per day, in sum $155 \mathrm{ml}$ D2 incorporated. 18 months break of 2 weeks, followed by 3 x 5 drops D2 per day.

19 months break.

20-24 months 6 x 6 drops D2 per day, in sum $180 \mathrm{ml}$ D2 incorporated.

24 months-today average dose 4 x 10 drops of D2 per day.

For monitoring of the therapy, the regular measurement of following parameter was arranged.

1. Lactatedehydrogenase (LDH) in serum. This enzyme is present in all cells, the occurrence in serum shows lysis of cells. LDH levels increase in all events resulting in cell degradation., i.e. myocardial or kidney infarction, embolism, autoimmunity, anemia, infectious diseases - or digestion of tumor cells.

2. Cell count of leukocytes p.m. for monitoring of tumor cell growth or cellular lysis or cellular migration activity.

3. Differential blood count for monitoring anemia appearance.

4. Liver enzymes gamma-glutamyltransferase (GGT), glutamate-oxalacetatetransaminase (GOT), glutamatepyruvate-transaminase (GPT), which occur in serum upon damage of liver cells, for monitoring possible intoxication of the liver.

Before the Amanita therapy, leukocyte levels in blood inceased continuously and exponentially, with a duplication time of about 21 months.

1-6 months of therapy: Initial dose at a cell count of $1,26 \times 10^{5} \mathrm{p} . \mathrm{m}$. is low, 3x15 drops of Amanita phalloides D4 per day. This leads to reduction of the growth rate of the cells (Figure 22). To stabilize and reduce the number of leukocytes, $1 \mathrm{ml}$ D2 i.e. 4 x 10 drops per day is applied. This leads to a reduction of leukocyte count.

7,5. months: At a cell count of $0,78 \times 10^{5}$ p.m., LDH increases revealing cell degradation. Cervical lymph nodes swell, fever and night sweat occur, hands and fingers are edemic, the patient is thirsty. The symptoms reveal lymphatic inflammation.

8 months: At a leukocyte cell count of $0,45 \times 10^{5}$ p.m., $\mathrm{LDH}$ is still high revealing lysis of cells. Fever and night sweat continue. Lymph nodes are shrunken. GPT value is higher, but still within the average range. GGT and GOT remain constant. Erythrocyte count is at $4,1 \times 10^{6} \mathrm{p} . \mathrm{m}$. Amanita uptake is interrupted.

A week later Leukocyte count is back to $1,08 \times 10^{5}$ p.m., erythrocyte count is low at $3,4 \times 10^{6}$ p.m..

9 months: Leukocyte level remains constant, LDH decreases, cell degradation ceases. The patient shows no symptoms any more. Erythrocyte count is back to normal range at $4,5 \times 10^{6} \mathrm{p} . \mathrm{m}$..

9 months until today - six years of stabilization: Amanita uptake interrupted by breaks lead to zigzag increase of leucocytes followed by a decrease coupled with the increase of LDH, i.e. cell degradation. Erythrocyte count remains around $4 \times 10^{6}$ p.m.. Reductions of leukocytes first occured with inflammation symptoms, later tumor cell digestions remained symptomless.

Liver enzymes remain in the average range all the time. Within the sixth year of therapy, thrombocyte levels decreased slowly to actual 130,000p.m. [minimal normal range is 150,000$]$.

\subsection{Therapy Example: Remission of a Tumor Disease}

Dilutions of Amanita phalloides are applied to a patient with two different tumors, rectum carcinome and thyroid cancer (Figure 23, [45]). The patient was born in 1930; she worked as soloist-singer in the theater; after marrying she became registrar. She studied music and eventually began teaching music at the Gymnasium (German prep school). Her whole life long she sang daily. During the war she smoked over a year and a half, up to 30 cigarettes a day, but gave it up because it damaged her singing voice. 
Her half-sister became ill with ovarian cancer and died at 46; her older sister was diagnosed with rectal cancer but lived to be 90 .

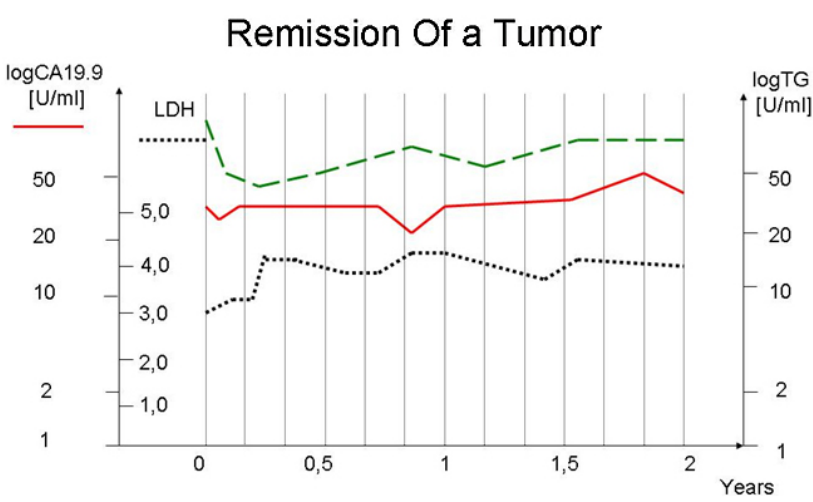

Figure 23. Remission of a patient with thyroid -CA and colon-CA (Explanation in main text)

The patient was diagnosed in 1995 with rectal cancer which was removed surgically. In 1999 recurrence occured that was treated operatively, and resulted in a stoma. No metastases and no lymph node involvement were found. She was treated with chemotherapy. Successive colonoscopies indicated ongoing polyp formation.

In March 2009 a thyroid cancer was diagnosed (pT3pNxcM0L1V1r0). After radiation therapy a tumor (50x38x42 mm) was surgically removed. The tumor tissue around the esophagus and trachea could not be completely removed. Radiation therapy followed in March, 2010, which could not completely remove the tumor tissue around the esophagus. A PET/CT scan showed a tumor in the jugular area $(37 \times 42 \times 36 \mathrm{~mm})$, and a prominent lymph node, left cervically (55 mm). A magnetic resonance imaging MRI in October 2010 confirmed recurrence. The patient was booked for a surgery, but hesitated.

The patient begins Amanita therapy in mid-October, 2010. At the beginning of the therapy she weighed $100 \mathrm{~kg}$ (220 lbs). The beginning dose is 2x5 drops of D2, until test results were obtained. $\mathrm{LDH}$ and the tumor markers CA19-9 (pancreas, gallbladder, intestine) and thyreoglobulin (TG, thyroid) are tracked. CA19-9 and TG are still both elevated, indicating that the rectal cancer from 1995 and the thyroid cancer from 2009 are both still active. Both tumor markes rised exponentially before Amanita therapy, indicating continuous growth of the tumor cells. The dose is increased to 2x10 drops of D2 per day. Figure 23 shows the activity of the tumor marker over the course of two years.

In December 2010 both markers show a change; LDH is slightly elevated, which indicates cell degradation, both tumor markers values decrease. Inflammation symptoms occur. After exponential growth before the Amanita therapy, this indicates effective cease of the tumor cell activity with Amanita. In January 2011 the patient notices brown sputum after taking $150 \mathrm{ml}$ of the D2. In March 2011, after taking 180ml D2, she notices yellow skin tone. The cysts in her larynx stabilize. The dose is lowered to $3 x 5$ drops of D2 per day. In May 2011, after uptake of 200ml D2, she experiences fever, vomiting, cough, and diarrhea. TG is slightly elevated, and the dose is adjusted to $4 \times 5$ drops D2 per day. In June 2011 tumor markers increase, leading to an elevated dose of 4x10 drops D2 per day. In July 2011 she suffers an ileus, which was relieved by endoscopy and enema. CT scan showed no tumor involvement. In October $2011500 \mathrm{ml}$ of D2 were taken, saliva is light brown. In December 2011 the patient suffers psychological crisis that needs no intervention. An MRI shows that the original tumor around the trachea disappeared (Figure 2).

During 2012 the patient continues uptake of 4x10 drops D2 daily, to further stabilize the tumor. In total, approximately 1.4 liters of D2 were taken within two years. In March 2012 the patient experiences a sticking pain in her throat, which radiates throughout her entire body and disappears within 10 minutes. In May 2012 she realizes that she could sing again, which she hadn't been able to do for years. In November 2012 bloody sputum occurs; she shows signs of a cold.

A colonoscopy from November 2012 shows disappearance of the polyps. Therefore the patient achieved a full remission. She interrupts the therapy for a week, both tumormarkers increase. She decides to continue Amanita therapy lifelong.

\subsection{Therapy Example: Prostate-Ca Stabilization}

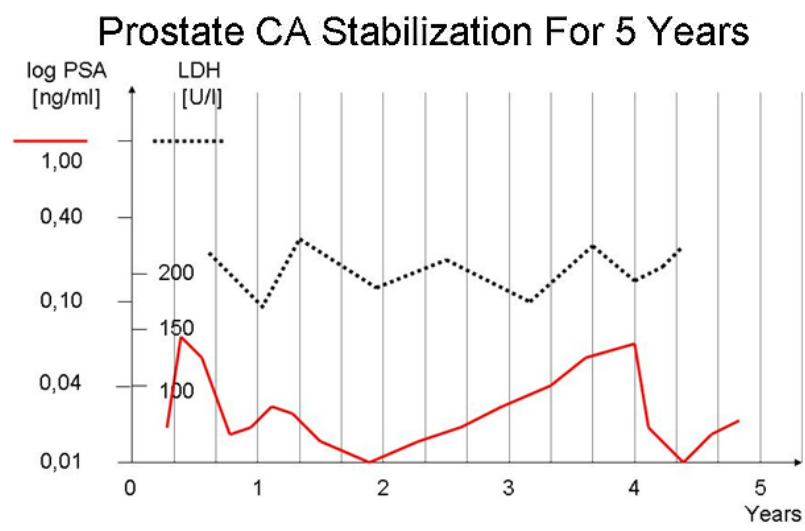

Figure 24. Prostata-CA stabilization over 5 years

Stabilization of a patient with pT4pN1M0R0, Gleason $9(4+5)$. At time of first diagnosis of prostate cancer PSA was at $13,0 \mathrm{ng} / \mathrm{ml}$. Natural duplication time of PSA is initially 1 month. Operation is impossible, infiltration of the right seminal ventricle and bladder were diagnosed. Therapy occurred with Tredantone and Proscar, in addition with Zometa. The initially fast growing tumor cells were halted with Proscar four months after first diagnosis. Amanita therapy started six months after first diagnosis (time 0 ), the patient decided to stop antihormone treatment. Initially the patient takes $50 \mathrm{ml}$ Amanita phalloides D2 in 2x10 drops per day. Further dosage of 3x15 drops of Amanita phalloides D4 per day was sufficient for stabilization for two years. Increasing PSA required a dosage of 3x15 drops of D3 per day. Further increase after nearly four years required the fast uptake of $100 \mathrm{ml} 22$ in $4 \times 10$ drops per day. This dosage reduces the PSA to a low amount. Further dosage is 10 drops of D2 per day. After a surgery of the knee, the patient pauses, resulting in increase of the PSA. This increase is slower than the initial exponential growth rate. At this time, MRI diagnoses complete remission. In case of natural duplication rate of PSA, the value would be over 500,000 ng/ml.

The patient was born in 1936 (Figure 24, [46]). Anamnesis finds familial risk, his grandfather had suffered from colon carcinoma. He has hypertension and neuropathies. First diagnosis of prostate-CA occurred in March 2007 by biopsy after micturition problems. No operation was possible due to infiltration to the bladder. XRay irradiation with 77 G-ray occurs in summer 2007. Anti-hormones Tredantone and Proscar were applied several times. In October 2008, the patient decides to stop 
anti-hormone treatment. He starts with Amanita phalloides D2 in a two months overlap with anti hormone therapy. Body weight is $82 \mathrm{~kg}$ and remains constant throughout the therapy. In addition to Amanita as the only tumor specific drug, the oral uptake of essential fatty acids and dermal application of Zinc salve is indicated.

For monitoring of the therapy, the regular measurement of following parameter is arranged

1. Lactatedehydrogenase (LDH) in serum.

2. Prostate Specific Antigen (PSA).

3. Cholesterol, differential blood count, liver transaminases to monitor general state of health.

A PSA value of $10 \mathrm{ng} / \mathrm{ml}$ is defined by academic medicine as intervention point. Below this value, watchful waiting is indicated. PSA values of the patient always remain below this point of interference. No other therapy is indicated. Initially, $50 \mathrm{ml}$ of D2 are sufficient to cease PSA values. With variant doses, the disease is stabilized for years. However, after five years a pause of the Amanita treatment leads to increasing PSA levels, tumor cells are still active. MRI diagnoses complete remission.

\subsection{Therapy Example: Exulcerating Mamma-Ca Stabilization}

The patient was born in 1954, no familial or other risk for tumor formation was found. Her body weight was 60 $\mathrm{kg}$ and stable throughout the therapy. Carcinoma was diagnosed in 1997 as calcification. In 2001 two tumors were found in the right breast. In 2006 the skin above one of the tumors started to open, and the ulceration continuously expanded. In 2008, the left breast was infiltrated. MRI pictures additionally diagnosed extensive infiltration of tissue into the thorax. Several skin metastases occured on the back thorax. The right lung wall collapsed. The skin over the right breast was open and bleeded continuously. The skin over the left breast started to open and bleeded. Axillar lymph nodes were swollen. Shortly before starting the Amanita therapy, the vertebral column was involved, leading to crocked position. Lymphatic oedema and ascites occured.

Operation was impossible, chemotherapy or radiation were not applied. Hyperthermic treatment was not successful.

\section{Exulcerating Mamma CA}

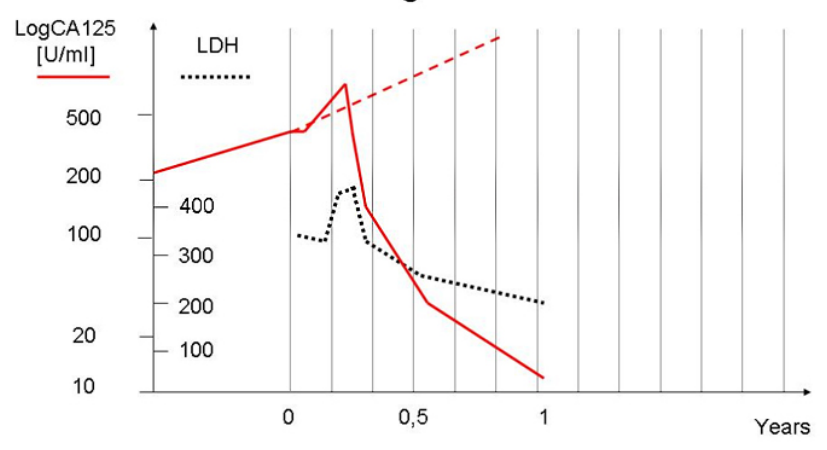

Figure 25. Rescue of a patient with exulcerating Mamma-CA (Explanation in main text)

Amanita treatment started beginning of 2009 (Figure 25, Time 0). As monitoring parameters were defined:

1. CA125 as tissue specific tumor marker,

2. $\mathrm{LDH}$ as degradation parameter and the clinical picture.

\section{Exulcerating Mamma CA}
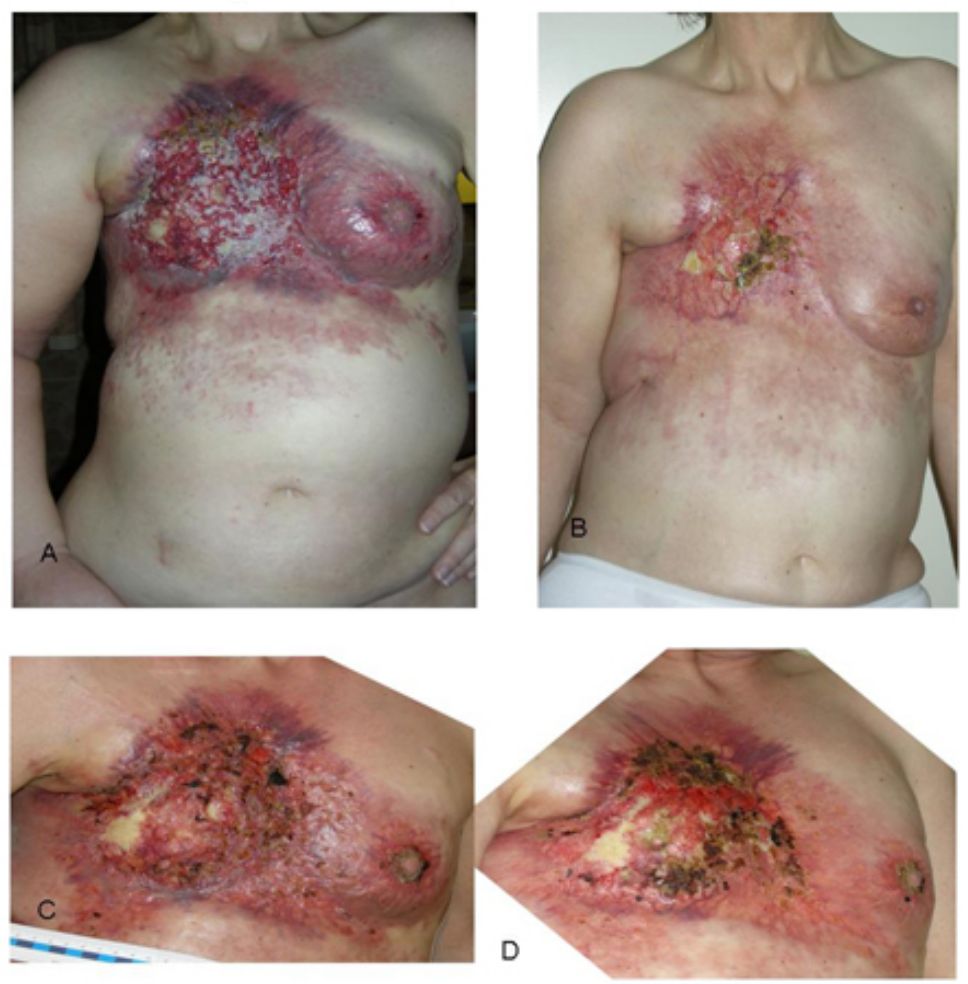

Figure 26. Exulcerating Mamma-CA: Clinical Picture (Explanation in main text)

CA125 and CA15.3 tumor markers rised exponentially before Amanita treatment. Continuous progression of the tumor occured, infiltration to adjacent tissues and metastazation continued. With a dosage of 4x10 drops Amanita phalloides D2 per day, CA125 and CA15.3 show no further increase, the tumor activity ceases, 2 weeks 
later tumor marke values remain constant. After two weeks, CA125 increases overexponentially, this is followed by an increase of $\mathrm{LDH}$, showing degradation of cells. The patient suffers from pain, lymph nodes swell and inflammation reaches the area of the ulcerating tissue. After 2,5 months, CA125 and LDH reach a maximum, followed by a decrease of both. The biochemical parameters are reflected in the clinical picture: Figure 26 shows the picture at the beginning of therapy (A), and after 4 months (B). The left breast becomes tumor free, the skin over the right part of thorax is closed. Two stages of the tumor degradation process after one (C) and three (D) months of Amanita therapy are shown. Black areas within the ulceration show nekrosis, digestion of tumor cells, biochemically visible as $\mathrm{LDH}$ increase and overexponential increase of tumor marker level due to destruction of tumor cells.

In parallel, biopsy revealed hormone dependent tumor cells. Therefore after 6 months of Amanita therapy, antihormone treatment followed to further stabilize the patient. Unfortunately, the occurence of the metastases in the back could not completely be hindered by further treatment, the patient decided end of 2011 for chemotherapy, and died during this treatment.

\section{Conclusion}

A tumor cell circumvents the restriction of the cell cycle. Mutations in proliferative genes are responsible: they allow replication, and lead to hyperplasic growth of tissue. Thus, a proliferative gene mutation is the primary initiative event in tumor formation. This finding is novel, so far researchers supported the thesis, tumor formation is due to a set of mutations in oncogenes and tumor suppressor genes. Latter gene families only add to differentiation patterns, but do not break the cell cycle. Neoplasms can be induced by a minimum of one dominant proliferative gene defect, two recessive proliferative gene defects, one proliferative gene defect over one oncogene defect, one proliferative gene defect over one tumor suppressor gene defect, or one proliferative gene defect over one deficiency [21].

Lack in differentiation provides a cell the competence to divide and migrate. Differentiation genes lead to determination of the tissue. Mutations of tld and $s p z$ in MBT and the oncogenic potential of pll and $\mathrm{Tl}$ alleles over MBT show that the determination process is destabilized in tumor formation. Mutation of tld in MBT is a secondary event: the addition of wild type tld in transgenic animals reduce vitality of MBT. Thus, tumor suppression is a secondary event, an adaptive mutation which suppresses lethality induced from a primary defect in a proliferative gene.

Mutations in human BRCA1 are responsible for about $10 \%$ of breast cancers and ovarian cancers. Its protein associates with Rad51, a member of a protein family mediating homologous pairing [47]. BRCA1 breast tumors are characterized by a high degree of genome plasticity. Proliferative genes in Drosophila induce somatic pairing gaps and replication initiation errors [12]. Accordingly, the phenotype of Drosophila reflects the molecular interaction of BRCA1: defect chromosome pairing and genome plasticity. Thus, BRCA might belong to the class of proliferative genes. All-1 transformations, responsible for human leukemia, allow replication and in thus All-1 is a proliferative gene.

Differentiation genes and proliferative genes interact genetically. The interactive potential has been used to identify the central interactors: switch genes. Their mutation induces lethality of tumor inducing gene combinations, but is permissive for wild type. Therefore, on the level of switch genes, tumor formation can be switched off. Switch genes are overexpressed and support tumor growth of cells. All of them are RNAPII transcription factors, therefore RNAPII should be fully used, represents one of the biochemical bottlenecks for growth of the tumor cell. Partial inhibition of RNAPII by amanitin in flies reduces activity of tumor cells. In humans, Amanita phalloides dilutions, containing amanitin, can be used for an effective tumor therapy.

Keep the tumor patient in life at life is the goal of a successful tumor therapy. With Amanita phalloides this wish reaches reality. At low cost, without any equipment the drug can be applied at home. In the classical sense Amanita is used as a remedy against fear of death. In the molecular sense this remedy was newly discovered as growth inhibitor specific for tumor cells.

Amanita therapy shows high potential for tumor treatment in all countries, even if monitoring of the patient is not possible. With the standard dose of $4 \times 10$ drops per day Amanita phalloides (zert. Riede) D2 [herbamed AG], fast growing and/or large tumor masses might be stabilized. This remains usually without side effects. The intake per linguale ensures a free way of the drug through the oral mucosa to the most metabolically active cells.

As the proliferative event and the induced switch gene overexpression are active in presumably any tumor cell in any tissue, Amanita can be applied to all types of tumors. Here some examples: leukemia, prostate or mamma carcinoma and rectal and thyroid tumor treatments are shown. Beyond the examples outlined here, experiences with more than 100 successful cases of therapy exist, thereunder a large variety of different tumors in different tissues. Allover, about $80 \%$ of the cases can be treated with success [48]. All non-responders are detected through appropriate monitoring within two months: after intake of $100 \mathrm{ml}$ Amanita phalloides D2, a change in the exponentially increasing tumor markers, and/or changes in the clinical picture should occur. Non-responders are sent to other therapeutic possibilities.

With amanitin, the activity of tumor cells is retarded in Drosophila. With Amanita phalloides the immune system is activated and digests tumor cells during an inflammation process in patients. This response to the Amanita therapy occurs in most of the cases, when $100 \mathrm{ml}$ of the D2 dilution is incorporated, inhibiting about $50 \%$ of all RNAPII activity in every cell. Inflammation is accompanied by tumor cell degradation and LDH increase. Without Amanita, the $\mathrm{LDH}$ levels remain within the normal range, the tumor grows, tumormarkers increase exponentially. Without treatment, the immune system does not efficiently digest tumor cells. Upon Amanita uptake, the immune system is able to attack the tumor. Its involvement shows that Amanita might not only interrupt the activity of tumor growth, but could alter antigen presentation, and thus immune cells might start to recognize tumor cells. 
Amanita therapy shows good results in therapy of a variety of tumor diseases. The therapy does not affect the activity of somatic cells. Thus dilutions from Amanita phalloides offer a strong tool for the future therapy of cancer.

\section{References}

[1] Jones DD 1974. Oncogene theory. Science. May 24;184(4139):853.

[2] Huebner RJ, Todaro GJ 1969. Oncogenes of RNA tumor viruses as determinants of cancer.Proc Natl Acad Sci U S A. Nov;64(3):1087-94. The genetics of colorectal cancer.

[3] Travali S, Koniecki J, Petralia S, Baserga R 1990. Oncogenes in growth and development. FASEB J. 4(14):3209-14.

[4] Klein G 1988. Oncogenes and tumor suppressor genes. Acta Oncol.;27(4):427-37.

[5] Weinberg RA 1996. How cancer arises. Sci Am. Sep;275(3):62-70.

[6] Weinberg RA1995. The molecular basis of oncogenes and tumor suppressor genes. Ann N Y Acad Sci. Jun 30;758:331-8.

[7] Foulds L 1958. The natural history of cancer. J Chronic Dis. Jul;8(1):2-37.

[8] Peto J, Doll R, Howard SV, Kinlen LJ, Lewinsohn HC 1977. A mortality study among workers in an English asbestos factory.Br J Ind Med. Aug;34(3):169-73.

[9] Kamb A 1994. Cancer. Sun protection factor p53 Nature. Dec 2229;372(6508):730-1.

[10] Kamb A, Gruis NA, Weaver-Feldhaus J, Liu Q, Harshman K, Tavtigian SV, Stockert E, Day RS 3rd, Johnson BE, Skolnick MH. 1994 A cell cycle regulator potentially involved in genesis of many tumor types. Science. Apr 15;264(5157):436-40.

[11] Nobori T, Miura K, Wu DJ, Lois A, Takabayashi K, Carson DA 1994. Deletions of the cyclin-dependent kinase-4 inhibitor gene in multiple human cancers. Nature. Apr 21;368(6473):753-6.

[12] Riede I (1997) Proliferative genes induce somatic pairing defects in Drosophila melanogaster and allow replication. Canc.Genet.Cytogenet. 97:143-154.

[13] Riede I 2012. Inhibition of Apoptosis in ALL-1 Leukemic Cell Lines: Allowance of Replication, constant Repair Replication, defect DNA Damage Control. J Cell Sci Ther 3:133.

[14] Anderson KV, Nüsslein-Volhard C 1984. Information for the dorsal--ventral pattern of the Drosophila embryo is stored as maternal mRNA. Nature. Sep 20-26;311(5983):223-7.

[15] Stein D, Nüsslein-Volhard C 1992. Multiple extracellular activities in Drosophila egg perivitelline fluid are required for establishment of embryonic dorsal-ventral polarity. Cell. Feb 7.

[16] Hashimoto C, Hudson KL, Anderson KV 1988. The Toll gene of Drosophila, required for dorsal-ventral embryonic polarity, appears to encode a transmembrane protein. Cell. Jan 29;52(2):269-79.

[17] Morisato D, Anderson KV 1994. The spätzle gene encodes a component of the extracellular signaling pathway establishing the dorsal-ventral pattern of the Drosophila embryo. Cell. Feb 25;76(4):677-88.

[18] Hecht PM, Anderson KV 1993. Genetic characterization of tube and pelle, genes required for signaling between Toll and dorsal in the specification of the dorsal-ventral pattern of the Drosophila embryo.Genetics. Oct;135(2):405-17.

[19] Shelton CA, Wasserman SA. 1993 pelle encodes a protein kinase required to establish dorsoventral polarity in the Drosophila embryo. Cell. Feb 26;72(4):515-25.

[20] Riede I 1996. Three mutant genes cooperatively induce brain tumor formation in Malignant Brain Tumor. Canc.Genet.Cytogenet. 90:135-141.

[21] Riede I 2002. Gene combinations inducing neoplasms in Drosophila. Drosophila Information Service 84: 103-114.

[22] Priyadarshini A, Chakraborti A, Mandal AK, Singh SK . 2013. Asp299Gly and Thr399Ile polymorphism of TLR-4 gene in patients with prostate cancer from North India. Indian J UrolJan;29(1):37-41.

[23] Salcedo R, Cataisson C, Hasan U, Yuspa SH, Trinchieri G. 2013. MyD88 and its divergent toll in carcinogenesis. Trends Immunol. May 6. pii: S1471-4906(13)00055-0.

[24] Xu Y, Xu Q, Yang L, Liu F, Ye X, Wu F, Ni S, Tan C, Cai G, Meng X, Cai S, Du X. 2013. Gene expression analysis of peripheral blood cells reveals toll-like receptor pathway deregulation in colorectal cancer.PLoS One. May 1;8(5):e62870.

[25] Shimell MJ, Ferguson EL, Childs SR, O'Connor MB1991. The Drosophila dorsal-ventral patterning gene tolloid is related to human bone morphogenetic protein 1. Cell. Nov 1;67(3):469-81.

[26] Childs SR, O'Connor MB. 1994 Two domains of the tolloid protein contribute to its unusual genetic interaction with decapentaplegic. Dev Biol. Mar;162(1):209-20.

[27] Massagué J. 1990. Transforming growth factor-alpha. A model for membrane-anchored growth factors. J Biol Chem. Dec 15;265(35):21393-6.

[28] Zhang N, Ye L, Wu L, Deng X, Yang Y, Jiang WG 2013. Expression of Bone Morphogenetic Protein-10 (BMP10) in Human Urothelial Cancer of the Bladder and its Effects on the Aggressiveness of Bladder Cancer Cells In Vitro. Anticancer Res. May;33(5):1917-25.

[29] Lee JH, Lee GT, Woo SH, Ha YS, Kwon SJ, Kim WJ, Kim IY 2013 Bone Morphogenetic Protein-6 in Renal Cell Carcinoma Promotes Tumor Proliferation via M2 Polarization of TumorInfiltrating Macrophages through Interleukin-10. Cancer Res. Apr 30.

[30] Li W, Cai HX, Ge XM, Li K, Xu WD, Shi WH 2013. Prognostic significance of BMP7 as an oncogene in hepatocellular carcinoma. Tumour Biol. Apr;34(2):669-74.

[31] Riede I 2000. Tumor inducing Drosophila: resistance to hydroxyurea and methane sulfonic acid methyl ester. Drosophila Information Service 83:140-144.

[32] Kowalczykowski SC 1991. Biochemical and biological function of Escherichia coli RecA protein: behavior of mutant RecA proteins. Biochimie. Feb-Mar;73(2-3):289-304.

[33] Kawabata M, Kawabata T, Nishibori M 2005. Role of recA/RAD51 family proteins in mammals. Acta Med Okayama. Feb;59(1):1-9.

[34] McKee BD, Ren X, Hong C 1996 A recA-like gene in Drosophila melanogaster that is expressed at high levels in female but not male meiotic tissues. Chromosoma. Apr;104(7):479-88.

[35] Canaani E, Nakamura T, Rozovskaia T, Smith ST, Mori T, et al. 2004. ALL- 1/MLL1, a homologue of Drosophila TRITHORAX, modifies chromatin and is directly involved in infant acute leukaemia. Br J Cancer 90: 756-760.

[36] Cimino G, Moir DT, Canaani O, Williams K, Crist WM, et al. 1991. Cloning of ALL-1, the locus involved in leukemias with the $\mathrm{t}(4 ; 11)(\mathrm{q} 21 ; \mathrm{q} 23), \quad \mathrm{t}(9 ; 11) \quad(\mathrm{p} 22 ; \mathrm{q} 23)$, and $\mathrm{t}(11 ; 19)(\mathrm{q} 23 ; \mathrm{p} 13)$ chromosome translocations. Cancer Res. 51: 6712-6714.

[37] Schichman SA, Canaani E, Croce CM (1995) Self-fusion of the ALL1 gene. A new genetic mechanism for acute leukemia. JAMA 273: 571-576.

[38] Gerschenson LE, Rotello RJ. 1992 Apoptosis: a different type of cell death. FASEB J. Apr;6(7):2450-5.

[39] Steller H, Grether ME 1994. Programmed cell death in Drosophila. Neuron. Dec;13(6):1269-74.

[40] Lakin ND and Jackson SP (1999) Regulation of p53 in response to DNA damage. Oncogene 18: 7644-7655.

[41] Riede I 2004. Switch Genes in Tumor Formation. Drosophila Information Service 87:54-59.

[42] Rozovskaia T, Feinstein E, Mor O, Foa R, Blechman J, Nakamura T, Croce CM, Cimino G, Canaani E 2001. Upregulation of Meis1 and HoxA9 in acute lymphocytic leukemias with the $\mathrm{t}(4: 11)$ abnormality. Oncogene Feb 15;20(7):874-8.

[43] Hudlebush HR, Lodahl M, Johnsen HE, Rasmussen T 2004. Expression of HOXA genes in patients with multiple myeloma Leuk.Lymphoma 45:1215-1217.

[44] Riede I 2010. Tumor Therapy with Amanita phalloides (Death Cap): Stabilization of B-Cell Chronic Lymphatic Leukemia. J. Altern. Complement. Med. 16:10, 1129-1132.

[45] Riede I 2013. Remission einer Tumorerkrankung mit der Amanita Therapie [Remission of a Tumor with the Amanita Therapy, in German] Naturheilpraxis 66:65-67.

[46] Riede I 2012. Tumor Therapy with Amanita phalloides (Death Cap): Long Term Stabilization of Prostate-Cancers. J Integr Oncol $1: 101$.

[47] Scully R, Chen J, Plug A et al. 1997. Association of BRCA1 with Rad 51 in mitotic and meiotic cells. Cell 88: 265-275.

[48] Riede I 2010. Erfahrungen mit der Amanita Therapie [Experiences with the amanita Therapy. In German] . Naturheilpraxis 63, 10701072 . 